\title{
A classification of inverse limit spaces of tent maps with periodic critical points
}

\author{
by
}

Lois Kailhofer (Milwaukee, WI)

\begin{abstract}
We work within the one-parameter family of symmetric tent maps, where the slope is the parameter. Given two such tent maps $f_{a}, f_{b}$ with periodic critical points, we show that the inverse limit spaces $\left(\mathbb{I}_{a}, f_{a}\right)$ and $\left(\mathbb{I}_{b}, g_{b}\right)$ are not homeomorphic when $a \neq b$. To obtain our result, we define topological substructures of a composant, called "wrapping points" and "gaps", and identify properties of these substructures preserved under a homeomorphism.
\end{abstract}

1. Introduction. Inverse limit spaces of one-dimensional maps commonly appear as attractors in dynamical systems $[1,2,6,9,14,21]$. This suggests the question: when are two inverse limit spaces of different onedimensional maps homeomorphic as topological spaces? One often studied family of one-dimensional maps is the one-parameter family of symmetric tent maps given by $f_{a}(x)=\min \{a x, a(1-x)\}$ for $x \in[0,1]$ and $a \in[1,2]$. Since any unimodal map without wandering intervals, restrictive intervals, or periodic attractors is conjugate to a tent map (see e.g. [16]), and as conjugate unimodal maps have homeomorphic inverse limit spaces, the family of tent maps is more inclusive than it seems at first glance. Given parameters $a \neq b$ it is unknown whether the corresponding inverse limit spaces $\left([0,1], f_{a}\right)$ and $\left([0,1], f_{b}\right)$ are homeomorphic as topological spaces, but partial results exist. Barge and Diamond [4] proved that for transitive Markov maps $f$ and $g$ of an interval $I$, if $(I, f)$ is homeomorphic to $(I, g)$ then the algebraic extensions $\mathbb{Q}(\alpha)=\mathbb{Q}(\beta)$ are equal, where $\alpha$ and $\beta$ are the spectral radii of the transition matrices for $f$ and $g$ respectively. However, it can be difficult to determine whether $\mathbb{Q}(\alpha)=\mathbb{Q}(\beta)$. Volkmer and Swanson [19] show that in the case when the characteristic polynomials of the transition matrices for $f$ and $g$ are irreducible, the discriminants of said characteristic polynomials are identical and $\mathbb{Z}(\alpha)=\mathbb{Z}(\beta)$. Most recently, Bruin [11] proved for $f_{a}$ and $f_{b}$ with finite critical orbits that if $\log (a)$ and $\log (b)$ are rationally

2000 Mathematics Subject Classification: Primary 54F15; Secondary 54H20, 58F03. 
independent, then the inverse limit spaces are not homeomorphic. There are other related results $[8,12,17,20]$.

In this paper we work with tent maps for which $a \in[\sqrt{2}, 2]$ and the turning point is periodic, i.e. letting $c$ denote the turning point, there is some positive integer $n$ such that $f_{a}^{n}(c)=c$. We show that the inverse limit spaces for any two such distinct maps are not homeomorphic. One can use [5, Theorem 2] to obtain results for $a \in[1, \sqrt{2}]$. Note that for $a \in[\sqrt{2}, 2], f_{a}$ is a transitive map [16]. One can construct non-transitive maps $f, g$ with $f$ not topologically conjugate to $g$, but $(\mathbb{I}, f)$ homeomorphic to $(\mathbb{I}, g)$.

Given a parameter $a \in[\sqrt{2}, 2]$ for which the turning point is periodic with period $n$, the kneading sequence of $f_{a}$ is given by the finite string of length $n, K\left(f_{a}\right)=b_{1}, \ldots, b_{n}$, where $b_{i}=R$ if $f_{a}^{i}(c)>c, b_{i}=L$ if $f_{a}^{i}(c)<c$, and $b_{n}=C$. Set $\mathbb{I}_{a}=\left[f_{a}^{2}(c), f_{a}(c)\right]$, the dynamical core of $f_{a}$. Define the inverse limit space $\left(\mathbb{I}_{a}, f_{a}\right)=\left\{\bar{x}=\left(x_{0}, x_{1}, \ldots\right) \mid \bar{x} \in \prod_{i=0}^{\infty} \mathbb{I}\right.$ and for all $\left.i>0, f_{a}\left(x_{i}\right)=x_{i-1}\right\}$. We now state our main result.

MaIN TheOREm. Let $f_{a}, f_{b}$ be tent maps with periodic turning points. If $K\left(f_{a}\right) \neq K\left(f_{b}\right)$ then $\left(\mathbb{I}_{a}, f_{a}\right)$ and $\left(\mathbb{I}_{b}, f_{b}\right)$ are not homeomorphic.

We obtain this result by building on the techniques and results in [15]. In Section 3, we give a brief outline of the definitions and results contained in [15]. Section 4 contains the proof of the main theorem. What follows is a brief synopsis of the proof of the main theorem.

Suppose $(\mathbb{I}, f)$ and $\left(\mathbb{I}^{\prime}, g\right)$ are homeomorphic via $h$. We first show that for sufficiently large $r$, there exists an $s$ such that each $r$-gap in $(\mathbb{I}, f$ ) (see Definition 31) will map under $h$ to exactly one $s$-gap in $(\mathbb{I}, g)$. Informally said, gaps go to gaps. We then show that when gaps go to gaps the kneading sequences of the bonding maps must be identical.

2. Preliminaries. In this section we make some general definitions and state some known results. The first half of this section applies to inverse limit spaces in general, and the second half is about inverse limit spaces of tent maps with periodic critical points. The omitted proofs can be found in [15].

Let $X$ be a topological space. If there exists a homeomorphism from $X$ onto $[0,1]$, then $X$ is called an arc. If there exists a continuous bijection from $[0,1)$ onto $X$, then $X$ is called a ray. The components of $X$ are the maximally connected subspaces of $X$. We define $\mathbb{N}=\{0,1, \ldots\}$ and $\mathbb{N}_{+}=\{1,2, \ldots\}$.

A continuum is a compact connected metric space. Let $x \in X$, a continuum. The composant of $x$ in $X$ is the union of all the proper subcontinua of $X$ that contain $x$. A subcontinuum $T$ of a space $X$ is an end continuum in $X$ if whenever $T \subseteq H, T \subseteq J$ for continua $H, J \subseteq X$, then either $H \subseteq J$ or $J \subseteq H$. The point $x \in X$ is an end point of $X$ if $\{x\}$ is an end continuum in $X$. For further discussion of continua, see [18]. 


\section{Lemma 1 [15, Lemma 1]. End points are topological invariants.}

Suppose that $\left\{X_{i}, d_{i}\right\}_{i=0}^{\infty}$ is a collection of compact metric spaces with $d_{i}$ bounded by 1 , and that for each $i, f_{i}: X_{i+1} \rightarrow X_{i}$ is a continuous map. The inverse limit space is given by

$$
\left\{X_{i}, f_{i}\right\}_{i=0}^{\infty} \equiv\left\{\bar{x}=\left(x_{0}, x_{1}, \ldots\right) \mid \bar{x} \in \prod_{i=0}^{\infty} X_{i}, f_{i}\left(x_{i+1}\right)=x_{i}, i \in \mathbb{N}\right\}
$$

and has metric $\bar{d}$ given by

$$
\bar{d}(\bar{x}, \bar{y})=\sum_{i=0}^{\infty} d_{i}\left(x_{i}, y_{i}\right) / 2^{i} .
$$

For each $i, \pi_{i}$ denotes the usual projection map from $\prod_{i=0}^{\infty} X_{i}$ into $X_{i}$. An inverse limit space $\left\{X_{i}, f_{i}\right\}_{i=0}^{\infty}$ is a continuum if $X_{i}$ is a continuum for every $i \in \mathbb{N}$ [18, Theorem 2.4]. If $X_{i}=X$ and $f_{i}=f$ for all $i$, the inverse limit space is denoted by $(X, f)$, and the map $\bar{f}:(X, f) \rightarrow(X, f)$ defined by $\bar{f}\left(\left(x_{0}, x_{1}, \ldots\right)\right)=\left(f\left(x_{0}\right), x_{0}, x_{1}, \ldots\right)$ is called the induced homeomorphism.

A continuous map $f:[a, b] \rightarrow[a, b]$ is called unimodal if there exists a unique turning or critical point, $c$, such that $f \mid[a, c]$ is increasing and $f \mid[c, b]$ is decreasing. For each $x \in[a, b]$, the itinerary of $x$ under the unimodal map $f$ is given by $I(x)=b_{0} b_{1} b_{2} \ldots$, where $b_{i}=R$ if $f^{i}(x)>c, b_{i}=L$ if $f^{i}(x)<c$, and $b_{i}=C$ if $f^{i}(x)=c$, with the usual convention that the itinerary stops after the first $C$. The kneading sequence of the unimodal map $f$, denoted $K(f)$, is defined to be the itinerary of $f(c)$. The parity-lexicographical ordering is put on the set of itineraries as follows. Set $L<C<R$. Let $W=w_{0} w_{1} \ldots$ and $V=v_{0} v_{1} \ldots$ be two distinct itineraries and let $k$ be the first index where the itineraries differ. If $k=0$, then $W<V$ if and only if $w_{0}<v_{0}$. If $k \geq 1$ and $w_{0} \ldots w_{k-1}=v_{0} \ldots v_{k-1}$ has an even number of $R$ 's, i.e., has even parity, then $W<V$ if and only if $w_{k}<v_{k}$; if $w_{0} \ldots w_{k-1}$ has an odd number of $R$ 's, then $W<V$ if and only if $v_{k}<w_{k}$. It is an elementary fact that the map $x \mapsto I(x)$ is monotone, i.e., $x<y$ implies that $I(x) \leq I(y)[13$, Lemma II.1.3]. Let $K(f)=a_{0} a_{1} \ldots$ Define the modified forward itinerary of $f(c)$, denoted $I^{\prime}(f(c))$, as follows: if $K(f)$ is infinite, let $I^{\prime}(f(c))=K(f)$; and if $K(f)$ has $n_{0}$ terms, then $I^{\prime}(f(c))=\left(a_{0} a_{1} \ldots a_{n_{0}-1}^{\prime}\right)^{\infty}$ where $a_{n_{0}-1}^{\prime} \in\{R, L\}$ is chosen such that $a_{0} a_{1} \ldots a_{n_{0}-1}^{\prime}<K(f)$.

Definition 2. Let $f: \mathbb{I} \rightarrow \mathbb{I}$ be a unimodal map. The backward itinerary of $\bar{x} \in(\mathbb{I}, f)$, denoted $B(\bar{x})=\left(b_{0}, b_{1}, b_{2}, \ldots\right)$, is a sequence of $R$ 's and $L$ 's such that

(i) $b_{i}=R$ if $x_{i} \geq c$ and $b_{i}=L$ if $x_{i} \leq c$,

(ii) if $x_{i}=c$ for some $i>0$, then $\left(b_{0}, b_{1}, \ldots, b_{i-1}\right)=\left(a_{i-1}, a_{i-2}, \ldots, a_{1}, a_{0}\right)$ where $I^{\prime}(f(c))=a_{0} a_{1} \ldots$ 
Define

$$
B_{f}=\{B(\bar{x}) \mid \bar{x} \in(\mathbb{I}, f)\} .
$$

Suppose that $c$ is periodic with period $n_{0}$, i.e. $n_{0}$ is the smallest integer such that $f^{n_{0}}(c)=c$. Let $\bar{x} \in(\mathbb{I}, f), B(\bar{x})=\left(b_{0}, b_{1}, b_{2}, \ldots\right)$, and $i<j \in \mathbb{N}$ be such that $x_{i}=c=x_{j}$. By the definition of backward itinerary, for every $k<i, b_{k}=a_{i-k-1}$ and $b_{k}=a_{j-k-1}$. However, $I^{\prime}(f(c))$ is periodic with period $n_{0}$, and hence for every $k<i, a_{i-k-1}=a_{j-k-1}$. Thus the notation $B(\bar{x})$ is well defined for every coordinate except $\max \left\{k \in \mathbb{N} \mid x_{k}=c\right\}$ where this maximum exists. Therefore, if $x_{i} \neq c$ for all $i \in \mathbb{N}$ or if $x_{i}=c$ for infinitely many $i \in \mathbb{N}$ then $\bar{x}$ has exactly one backward itinerary and if $x_{i}=c$ for finitely many $i \in \mathbb{N}$ then $\bar{x}$ has exactly two backward itineraries that differ in exactly one coordinate, $\max \left\{i \in \mathbb{N} \mid x_{i}=c\right\}$. For further discussion of itineraries see $[9,10]$.

The one-parameter family of tent maps is given by $f_{a}(x) \equiv \min \{a x$, $a(1-x)\}$ for $x \in[0,1]$ and $a \in[\sqrt{2}, 2]$. Note that $f_{a}$ is unimodal for all $a \in[\sqrt{2}, 2]$. Let $\mathbb{I}_{a} \equiv\left[f_{a}^{2}(c), f_{a}(c)\right]$. Notice that the interval $\mathbb{I}_{a}$ is invariant under $f_{a}$ and $f_{a}$ is locally eventually onto on $\mathbb{I}_{a}$; a map $g: I \rightarrow I$ is locally eventually onto (l.e.o.) if for every non-degenerate interval $J \subset I$ there exists an $n>0$ such that $g^{n}(J)=I$. The interval $\mathbb{I}_{a}$ is called the core of the map $f_{a}$. The inverse limit space $\left([0,1], f_{a}\right)$ is identical to $\left(\mathbb{I}_{a}, f_{a}\right)$ except possibly for an additional ray entwined with $\left(\mathbb{I}_{a}, f_{a}\right)$. Clearly any subcontinuum of $\left(\mathbb{I}_{a}, f_{a}\right)$ is contained in $\left([0,1], f_{a}\right)$ since $\mathbb{I}_{a} \subset[0,1]$; the converse is not necessarily true. For the rest of this paper, $f_{a}$ is assumed to be restricted to its core.

3. Previous results. In this section we give a brief synopsis of the definitions and results contained in [15]. No proofs are included in the interest of brevity.

Fix $a \in[\sqrt{2}, 2]$ such that $c$ is periodic with period $n_{0}$, i.e. $n_{0}$ is the smallest positive integer such that $f_{a}^{n_{0}}(c)=c$. For ease of notation, set $\mathbb{I} \equiv \mathbb{I}_{a}, f \equiv f_{a} \mid \mathbb{I}, c_{i} \equiv f^{i}(c), \mathbb{I}_{R} \equiv\left[c, c_{1}\right]$, and $\mathbb{I}_{L} \equiv\left[c_{2}, c\right]$. Except at its end points, $(\mathbb{I}, f)$ is everywhere locally homeomorphic with the product of a Cantor set and an arc [3]. The composant of $\bar{x} \in(\mathbb{I}, f)$ is the set of all points $\bar{y} \in(\mathbb{I}, f)$ for which $B(\bar{y})$ is eventually identical to $B(\bar{x})$, i.e. if $B(\bar{x})=\left(b_{0}, b_{1}, \ldots\right)$ and $B(\bar{y})=\left(d_{1}, d_{2}, \ldots\right)$ then $\bar{y}$ is in the composant of $\bar{x}$ if and only if there exists an $N \in \mathbb{N}$ such that for all $n \geq N, b_{n}=d_{n}$ [9, Corollary 2.10].

Definition 3. Let $w=\left(w_{0}, w_{1}, \ldots\right) \in B_{f}$. Define

$$
\mathcal{A}_{w}=\left\{\bar{x} \in(\mathbb{I}, f) \mid \pi_{i}(\bar{x}) \in \mathbb{I}_{w_{i}} \text { for all } i \in \mathbb{N}\right\},
$$

and

$$
\mathcal{A}_{w}^{n}=\bar{f}^{n}\left(\mathcal{A}_{w}\right) \quad \text { for all } n \in \mathbb{N} \text {. }
$$


Note that for all $n \in \mathbb{N}$ and $w \in B_{f}, \mathcal{A}_{w}^{n}=\bigcup\left\{\mathcal{A}_{v} \mid \mathcal{A}_{v} \subset \mathcal{A}_{w}^{n}\right\}$. Each $\mathcal{A}_{w}$ is a non-degenerate arc. If $c$ were not periodic, then $\mathcal{A}_{w}$ could be degenerate.

Lemma 4 [15, Lemma 4, Corollary 5, and Lemma 6]. Let $w \in B_{f}$. For each $n \in \mathbb{N}, \pi_{n} \mid \mathcal{A}_{w}^{n}$ is a homeomorphism, $\pi_{n}\left(\mathcal{A}_{w}^{n}\right)=\pi_{0}\left(\mathcal{A}_{w}\right)$, and the interval $\pi_{n}\left(\mathcal{A}_{w}^{n}\right)$ is completely determined by the first $n_{0}+1$ elements of $w$.

Lemma 4 allows us to easily graph the map $\pi_{n}\left(\mathcal{A}_{w}^{n}\right)$ for any $n \in \mathbb{N}$ and $w \in B_{f}$ by looking at the graph of $f^{n_{0}}$.

EXAMPLE 1.1. Let $\Upsilon$ be the tent map with kneading sequence $R L R R C$. Consider $\Upsilon^{5}$ (Figure 1). The bottom axis is partitioned by the interval itineraries of length 6 allowed for $\Upsilon$. Thus if $w \in B_{\Upsilon}$ the first 6 elements of $w$ will match one of these itineraries, and $\pi_{n}\left(A_{w}^{n}\right)$ will then be the same as $\Upsilon^{5}$ applied to the interval that corresponds to that itinerary. For example, if $w=R L R L R R \ldots$ then $\pi_{n}\left(A_{w}^{n}\right)=\left[c, c_{4}\right]$.

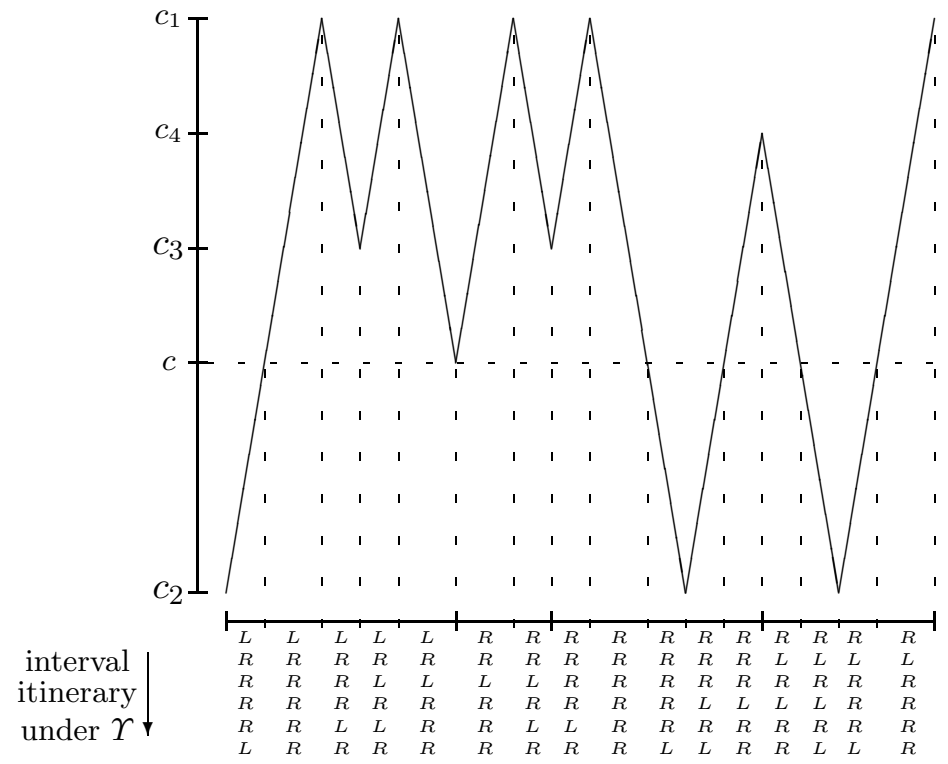

Fig. 1. The fifth iterate of $\Upsilon$

DEFINITION 5. Let

$$
\bar{c}=\left(c, c_{n_{0}-1}, c_{n_{0}-2}, \ldots, c_{2}, c_{1}\right)^{\infty}
$$

and

$$
\bar{c}_{i}=\bar{f}^{i}(\bar{c}) \quad \text { for all } i \in \mathbb{N} .
$$

Lemma 6 [7]. The end points of $(\mathbb{I}, f)$ are $\bar{c}, \bar{c}_{1}, \ldots, \bar{c}_{n_{0}-1}$.

As the number of end points in $(\mathbb{I}, f)$ is the period of the critical point of $f$, to prove our main result, we need only consider tent maps whose critical 
points have the same period. Fix $b \neq a \in[\sqrt{2}, 2]$ such that $c$ has period $n_{0}$ under $f_{b}$. Let $\mathbb{I}^{\prime} \equiv\left[f_{b}^{2}(c), f_{b}^{1}\left(c_{1}\right)\right]$ and for ease of notation, set $g \equiv f_{b} \mid \mathbb{I}^{\prime}$, $\left.c_{i}^{\prime} \equiv g^{i}(c)\right), \mathbb{I}_{L}^{\prime} \equiv\left[c_{2}^{\prime}, c\right]$, and $\mathbb{I}_{R}^{\prime} \equiv\left[c, c_{1}^{\prime}\right]$. In the interests of brevity, when we define a structure in $(\mathbb{I}, f)$, we will assume that a similar definition has been made for $\left(\mathbb{I}^{\prime}, g\right)$. To help distinguish to which space the structure belongs, we put a prime after the notation for the structures of $\left(\mathbb{I}^{\prime}, g\right)$. Thus, for example, as the core of $f$ is denoted by $\mathbb{I}$, the core of $g$ is denoted by $\mathbb{I}^{\prime}$.

Definition 7. Let $U=\left\{u_{i}\right\}_{i=1}^{n}$ be an open cover of a topological space $X$. The set $U$ is a chaining of $X$ provided $u_{i} \cap u_{j} \neq \emptyset$ if and only if $|i-j| \leq 1$. The $u_{i}$ 's are called the links of the chain.

DEFINITION 8. Let $U=\left\{u_{i}\right\}_{i=1}^{n}$ and $V=\left\{v_{i}\right\}_{i=1}^{m}$ be chainings of a topological space. The set $V$ is said to refine $U, V \prec U$, if for every $1 \leq i \leq m$ there exists $1 \leq j \leq n$ such that $v_{i} \subset u_{j}$.

Assume that $\left(\mathbb{I}^{\prime}, g\right)$ and $(\mathbb{I}, f)$ are homeomorphic and $h:\left(\mathbb{I}^{\prime}, g\right) \rightarrow(\mathbb{I}, f)$ is a homeomorphism. We know that end points are topologically invariant, thus we define a family of chainings on $\left(\mathbb{I}^{\prime}, g\right)$ and $(\mathbb{I}, f)$ to exploit this fact. We first define a family of chainings on $\mathbb{I}$.

Definition 9. Let $\left\{\mathrm{L}_{p, m} \mid p, m \in \mathbb{N}\right\}$ be a set of chainings for $\mathbb{I}$ with the following properties:

(i) $\mathrm{L}_{p, m} \prec \mathrm{L}_{i, j}$ if $p \geq i$ and $m \geq j$,

(ii) for all $p, m \in \mathbb{N}$ and $0 \leq i<n_{0}, c_{i}$ is contained in exactly one link of $\mathrm{L}_{p, m}$,

(iii) for all $p, m \in \mathbb{N}$, if $\ell \in \mathrm{L}_{p+1, m}$ then there exists $\gamma \in \mathrm{L}_{p, m}$ such that $f(\ell) \subset \gamma$,

(iv) if $L$ is a chaining of $\mathbb{I}$ there exist $r, s \in \mathbb{N}$ such that $\mathrm{L}_{r, s} \prec L$.

Examples of such chainings are constructed in [15]. We then take the inverse image of this chaining under the projection map to get our family of chainings of $(\mathbb{I}, f)$.

Definition 10. For all $p, m \in \mathbb{N}$, let $\mathcal{L}_{p, m}=\pi_{p}^{-1}\left(\mathrm{~L}_{p, m}\right)$.

The subscript $p$ refers to the coordinate of the projection map used and the subscript $m$ refers to the fineness of the mesh of the chaining on $\mathbb{I}$. As a direct result of its construction, this family of chainings has the following properties.

Lemma 11 [15, Lemma 16]. Fix $p, m, q, j \in \mathbb{N}$. The following hold:

(i) $\mathcal{L}_{p, m} \prec \mathcal{L}_{q, j}$ if $p \geq q$ and $m \geq j$,

(ii) for every $0 \leq i<n_{0}$, there exists a unique $\ell \in \mathcal{L}_{p, m}$ such that $\bar{c}_{i} \in \ell$,

(iii) if $L$ is a chaining of $(\mathbb{I}, f)$ there exist $p, m \in \mathbb{N}$ such that $\mathcal{L}_{p, m} \prec L$. 
Definition 12. Fix $m, p \in \mathbb{N}$. For each $0 \leq j<n_{0}$ define $\ell_{p, m}^{c_{j}}$ to be the link of $\mathcal{L}_{p n_{0}, m}$ that contains the end point $\bar{c}_{j}$.

Fix $p, m \in \mathbb{N}$. There exist, by Lemma $11\left(\right.$ iii), $q, r \in \mathbb{N}$ such that $h\left(\mathcal{L}_{q n_{0}, r}^{\prime}\right)$ $\prec \mathcal{L}_{p n_{0}, m}$ and hence the homeomorphic image of the links of $\mathcal{L}_{q n_{0}, r}^{\prime}$ that contain end points is contained in the links of $\mathcal{L}_{p n_{0}, m}$ that contain end points, symbolically $h\left(\bigcup_{i=0}^{n_{0}-1} \ell_{q, r}^{c_{i}^{\prime}}\right) \subset \bigcup_{i=0}^{n_{0}-1} \ell_{p, m}^{c_{i}}$. We now compare how the composants of $(\mathbb{I}, f)$ pass through the links of $\mathcal{L}_{p n_{0}, m}$ that contain end points with how the composants of $\left(\mathbb{I}^{\prime}, g\right)$ pass through the links of $\mathcal{L}_{q n_{0}, r}^{\prime}$ that contain end points. To simplify this comparison, we restrict our attention to the composant of $\left(\mathbb{I}^{\prime}, g\right)$ that contains $\bar{c}^{\prime}$ and its image under the homeomorphism. We label this composant $\mathcal{C}^{\prime}$. We know that $h\left(\bar{c}^{\prime}\right)$ is one of the end points of $(\mathbb{I}, f)$, but do not know which one. Choose $i \in \mathbb{N}$ such that $f^{i} \circ h\left(\bar{c}^{\prime}\right)=\bar{c}$, and set $\bar{h} \equiv \bar{f}^{i} \circ h$. Thus $\bar{h}\left(\mathcal{C}^{\prime}\right) \rightarrow \mathcal{C}$.

Lemma 13 [15, Lemma 19]. The composant $\mathcal{C}$ is a ray with end point $\bar{c}$.

EXAMPLE 1.2. Let $\Upsilon$ be the tent map with kneading sequence $R L R R C$. As the chaining $\mathcal{L}_{p 5, m}$ was defined using $\pi_{p 5}^{-1}$ on a chaining of $\mathbb{I}$, we can see how $\mathcal{C}$ passes through the links of $\mathcal{L}_{p 5, m}$ by examining the map $\pi_{p 5}$ : $\mathcal{C} \rightarrow \mathbb{I}$. Let $\bar{c} \in \mathcal{A}_{w}^{p 5}$. We know $w=R R L R R R \ldots$, thus $\pi_{p 5}\left(\mathcal{A}_{w}^{p 5}\right)=\left[c, c_{1}\right]$ (Lemma 4). Furthermore, as $c$ has a period of 5 under $f, \bar{c} \in \mathcal{A}_{w}^{(p+1) 5}$ and $\pi_{(p+1) 5}\left(\mathcal{A}_{w}^{(p+1) 5}\right)=\left[c, c_{1}\right]$. Thus $\pi_{p 5}\left(\mathcal{A}_{w}^{(p+1) 5}\right)=f^{5} \circ \pi_{(p+1) 5}\left(\mathcal{A}_{w}^{(p+1) 5}\right)=$ $f^{5}\left(\left[c, c_{1}\right]\right)$. See Figures 2 and 1 . As there was nothing special about our choice of $p$, we know $\pi_{(p+1) 5}(\mathcal{C})$ has the same basic "shape" as $\pi_{p 5}(\mathcal{C})$. Thus we can find the next "part" of $\pi_{p 5}(\mathcal{C})$ by finding $f^{5}\left(\left[c_{1}, c_{3}\right]\right)$. Again see Figures 2 and 1 . By proceeding in this manner, we can graph $\pi_{p 5}: \mathcal{C} \rightarrow \mathbb{I}$.

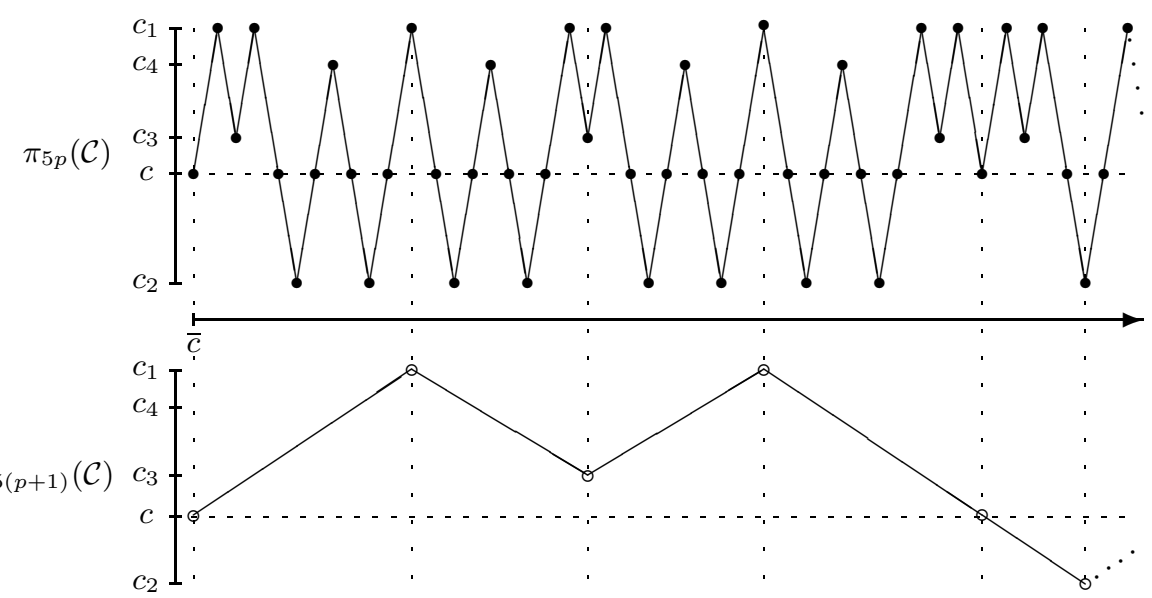

Fig. 2. The projections of the $p$-wrapping points $(\bullet)$ and the $(p+1)$-wrapping points $(\circ)$ 
Definition 14. Let $\gamma:[0,1) \rightarrow \mathcal{C}$ be a continuous bijection. Define the $\mathcal{C}$-induced order on $\mathcal{C}, \triangleleft$, by $\bar{x} \triangleleft \bar{y}$ if and only if $\gamma^{-1}(\bar{x})<\gamma^{-1}(\bar{y})$.

We now define a set of $p$-special points that indicate when $\mathcal{C}$ passes through a link of $\mathcal{L}_{p n_{0}, m}$ that contains an end point. These $p$-special points have a natural one-to-one correspondence with the components of $\mathcal{C}$ intersected with the links of $\mathcal{L}_{p n_{0}, m}$ that contain an end point (Proposition 16). When ordered, the $p$-special points identify how $\mathcal{C}$ passes through the links of $\mathcal{L}_{p n_{0}, m}$. We can then compare the special points of $\mathcal{C}$ to the special points of $\mathcal{C}^{\prime}$.

Definition 15. Fix $p, j \in \mathbb{N}, 0 \leq j<n_{0}$. Let

$$
\widetilde{\Phi}_{p, j}=\left\{\bar{x} \in \mathcal{C} \mid \pi_{p n_{0}}(\bar{x})=c_{j}\right\}, \quad \widetilde{\Phi}_{p}=\bigcup_{i=0}^{n_{0}-1} \widetilde{\Phi}_{p, i} .
$$

If $\bar{x} \in \widetilde{\Phi}_{p}$ it is called a $p$-special point. Define $\Phi_{p, j}$ to be the sequence of the elements of $\widetilde{\Phi}_{p, j}$ in $\mathcal{C}$-induced order and $\Phi_{p}$ to be the sequence of the elements of $\widetilde{\Phi}_{p}$ in $\mathcal{C}$-induced order.

Note that if $u<p \in \mathbb{N}$, then $\widetilde{\Phi}_{u} \supset \widetilde{\Phi}_{p}$.

EXAMPLE 1.3. Let $\Upsilon$ be the tent map with kneading sequence $R L R R C$. Figure 3 shows the $p$-special points of $\mathcal{C}$. As stated in Definition 15, $p$-special points are the points of $\mathcal{C}$ which the projection $\pi_{p 5}$ takes to $c_{i}$ for some $0 \leq i<5$.

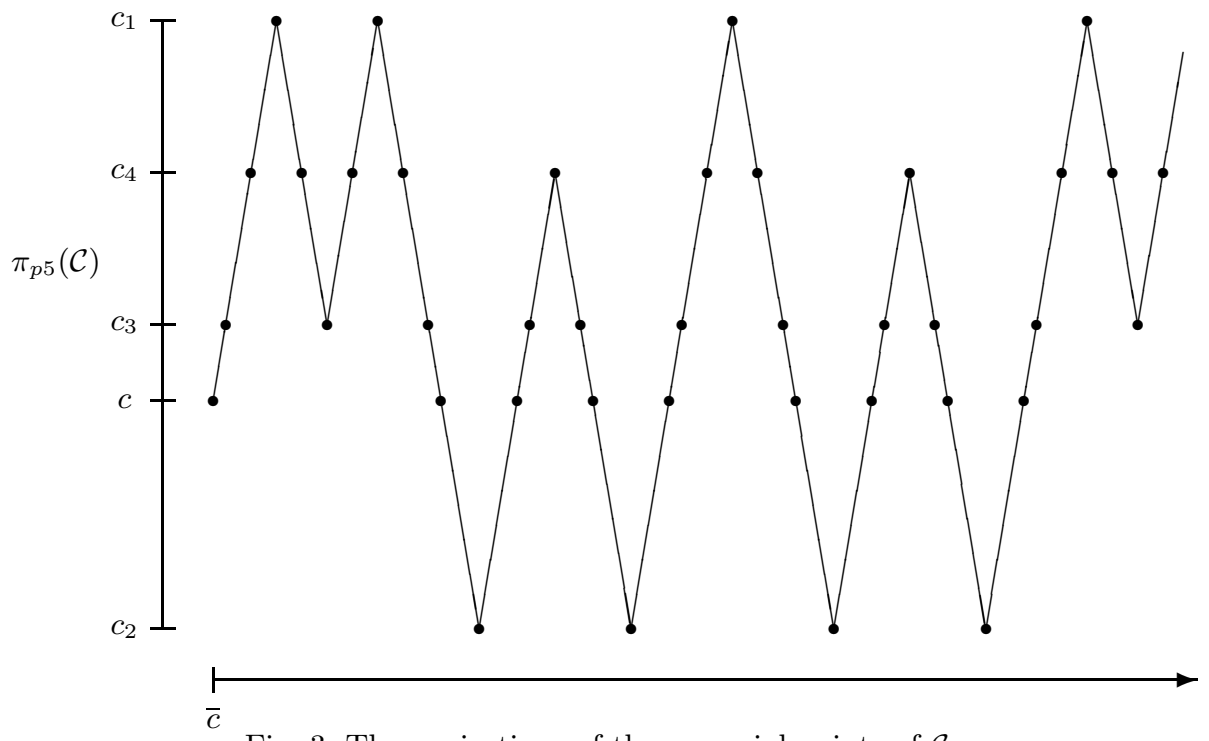

Fig. 3. The projections of the $p$-special points of $\mathcal{C}$ 
Proposition 16 [15, Proposition 25]. Fix $p, m, k \in \mathbb{N}, 0 \leq k<n_{0}$. Let $D$ be a component of $\mathcal{C} \cap \ell_{p, m}^{c_{k}}$. The closure of $D$ is an arc, and $D$ contains exactly one element of $\widetilde{\Phi}_{p, k}$.

We cannot guarantee that the homeomorphism $h$ takes special points to special points. We therefore define a "fudged" map $\bar{h}_{q, p}$ such that $\bar{h}_{q, p}\left(\widetilde{\Phi}_{q}^{\prime}\right)$ $\subset \widetilde{\Phi}_{p}$.

Definition 17. Fix $m, p, q, r \in \mathbb{N}$ such that $\bar{h}\left(\mathcal{L}_{q, r}^{\prime}\right) \prec \mathcal{L}_{p, m}$. Define $\bar{h}_{q, p}: \widetilde{\Phi}_{q}^{\prime} \rightarrow \widetilde{\Phi}_{p}$ as follows. If $\bar{w}^{\prime} \in \widetilde{\Phi}_{q, j}^{\prime}$ and $\bar{h}\left(\bar{c}_{j}^{\prime}\right)=\bar{c}_{i}$, then $\bar{h}_{q, p}\left(\bar{w}^{\prime}\right)$ is the element of $\widetilde{\Phi}_{p, i}$ that lies on the same component of $\ell_{p, m}^{c_{i}}$ as $\bar{h}\left(\bar{w}^{\prime}\right)$.

The map $\bar{h}_{q, p}$ is one-to-one, but not necessarily onto. Hence, let $i_{0}, i_{1}$, $i_{2}, \ldots$ be such that

$$
\bar{h}_{q, p}\left(\Phi_{q}^{\prime}\right)=\left\{\bar{h}_{q, p}\left(\bar{w}_{0}^{\prime}\right), \bar{h}_{q, p}\left(\bar{w}_{1}^{\prime}\right), \ldots\right\}=\left\{\bar{w}_{i_{0}}, \bar{w}_{i_{1}}, \bar{w}_{i_{2}}, \ldots\right\} \subset \Phi_{p, 0} .
$$

The sequence $i_{0}, i_{1}, i_{2}, \ldots$ is predictable, it has a pattern. Unfortunately, this pattern is too complicated to predict without a better understanding of the behavior of $\mathcal{C}$. Hence, for each $p \in \mathbb{N}$, we identify the set of points, $p$-wrapping points, at which the projection $\pi_{p n_{0}}$ of $\mathcal{C}$ turns or wraps (Definition 18). For technical reasons, we include those points of $\mathcal{C}$ that map to $c$ under $\pi_{p n_{0}}$ in the set of $p$-wrapping points. The set of $p$-wrapping points is a proper subset of the set of $p$-special points and the $p$-special points are a proper subset of the $(p-1)$-wrapping points.

Definition 18. For each $p \in \mathbb{N}$, define

$$
\widetilde{\mathrm{W}}_{p}=\left\{\bar{x} \in \mathcal{C} \mid \bar{x} \in \mathcal{A}_{v}^{p n_{0}} \cap \mathcal{A}_{w}^{p n_{0}}, v \neq w \in B_{f}\right\} \cup\{\bar{c}\} .
$$

If $\bar{x} \in \widetilde{\mathrm{W}}_{p}$ then $\bar{x}$ is called a $p$-wrapping point. Define $\mathrm{W}_{p}$ to be the sequence of all $p$-wrapping points in $\mathcal{C}$-induced order.

EXAMPLE 1.4. Let $\Upsilon$ be the tent map with kneading sequence $R L R R C$. Figure 2 shows the $p$-wrapping points and the $(p+1)$-wrapping points of $\mathcal{C}$. Note that $\widetilde{\mathrm{W}}_{p+1} \subset \widetilde{\mathrm{W}}_{p}$.

Definition 19. Let $\bar{x} \in \widetilde{\mathrm{W}}_{p} \backslash\{\bar{c}\}$, and let $v \neq w \in B_{f}$ be such that $\bar{x} \in \mathcal{A}_{v}^{p n_{0}} \cap \mathcal{A}_{w}^{p n_{0}}$. By Definition 2, $v$ and $w$ differ in exactly one coordinate, say $k$. Define the $p$-level of $\bar{x}$ and $\bar{c}$ by $L_{p}(\bar{x})=k$ and $L_{p}(\bar{c})=\infty$.

Suppose $\bar{x} \in \widetilde{\mathrm{W}}_{p}, \bar{x} \neq \bar{c}$. The point $\bar{x}$ has exactly 2 backward itineraries. These itineraries differ in only one coordinate, the highest coordinate of $\bar{x}$ that is $c$, say $j$. The $p$-level of $\bar{x}$ is then $j-p n_{0}$ as the backward itineraries $v$ and $w$ used in Definition 19 start being compared to $\bar{x}$ in the $p n_{0}$ coordinate of $\bar{x}$. Figure 4 shows the $p$-wrapping points with their $p$-levels. The set $\left\{L_{p}(\bar{w}) \mid \bar{w} \in \widetilde{\mathrm{W}}_{p} \backslash\{\bar{c}\}\right\}$ is unbounded. 


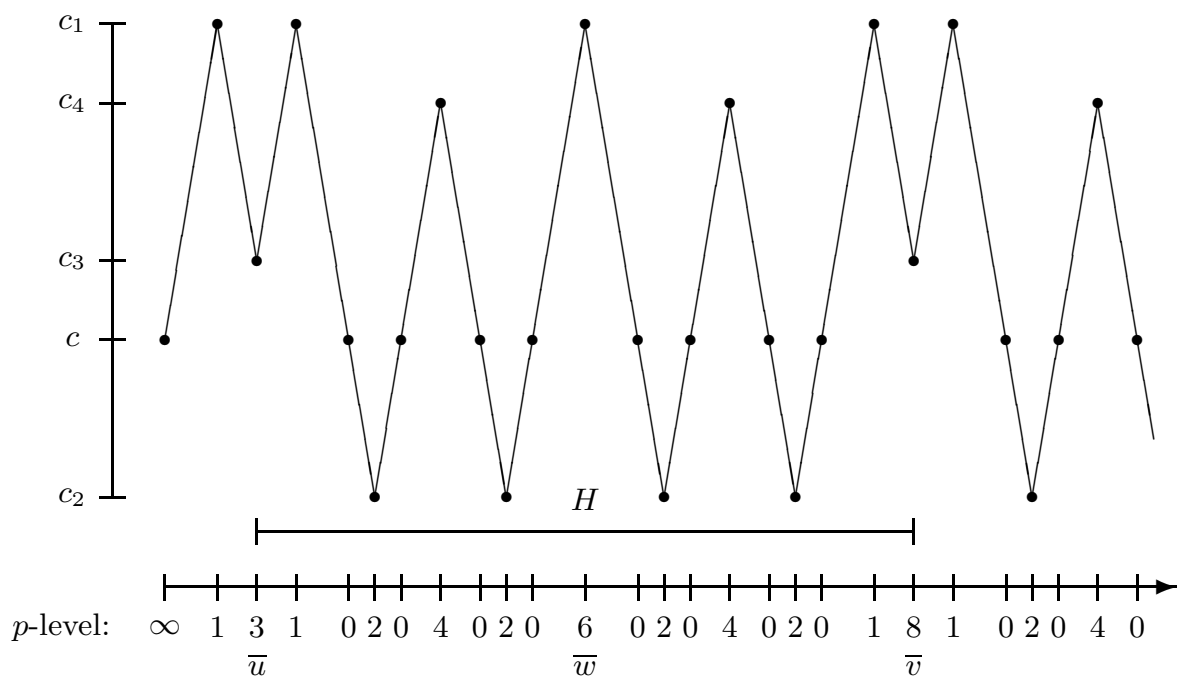

Fig. 4. The map $\pi_{5 p}: \mathcal{C} \rightarrow \mathbb{I}$, the $p$-wrapping points with their $p$-levels, and an example of $\bar{w}$ and $H$ that satisfy the hypotheses of Proposition 23

Definition 20. Fix $p \in \mathbb{N}$. Let $H \subset \mathcal{C}$ be an arc, $\stackrel{\circ}{H} \cap \mathrm{w}_{p}=\left\{\bar{h}_{1}, \ldots, \bar{h}_{n-1}\right\}$ and $\partial H=\left\{\bar{h}_{0}, \bar{h}_{n}\right\}$. The arc $H$ is p-symmetric if $\pi_{p n_{0}}\left(\bar{h}_{0}\right)=\pi_{p n_{0}}\left(\bar{h}_{n}\right)$ and for each $0<i<n, L_{p}\left(\bar{h}_{i}\right)=L_{p}\left(\bar{h}_{n-i}\right)$. The arc $H$ is p-pseudosymmetric if for each $0 \leq i \leq n, \pi_{p n_{0}}\left(\bar{h}_{i}\right)=\pi_{p n_{0}}\left(\bar{h}_{n-i}\right)$. If $H$ is $p$-symmetric or $p$ pseudosymmetric, $n$ must be even; thus the center of $H$, denoted $\bar{\kappa}_{H}$, is the point $\bar{h}_{n / 2}$.

Let $\bar{y}, \bar{x} \in \widetilde{\mathrm{W}}_{p}$. By the definition of $p$-level, we know that if $L_{p}(\bar{y})=L_{p}(\bar{x})$, then $\pi_{p n_{0}}(\bar{y})=\pi_{p n_{0}}(\bar{x})$. Thus all $p$-symmetric arcs are also $p$-pseudosymmetric. However, as $L_{p}(\bar{y}) \bmod n_{0}=L_{p}(\bar{x}) \bmod n_{0}$ implies that $\pi_{p n_{0}}(\bar{y})=$ $\pi_{p n_{0}}(\bar{x})$, not all $p$-pseudosymmetric arcs are $p$-symmetric.

Definition 21. Let $p \in \mathbb{N}$. Let $H, G \subset \mathcal{C}$ be arcs. Set

$$
\stackrel{\circ}{G} \cap \mathrm{W}_{p}=\left\{\bar{g}_{1}, \ldots, \bar{g}_{n-1}\right\}, \quad \partial G=\left\{\bar{g}_{0}, \bar{g}_{n}\right\}, \quad \stackrel{\circ}{H} \cap \mathrm{W}_{q}=\left\{\bar{h}_{1}, \ldots, \bar{h}_{m-1}\right\}
$$

and $\partial H=\left\{\bar{h}_{0}, \bar{h}_{m}\right\}$. The arcs $G$ and $H$ are said to have the same $p$-shape if $n=m$ and $\pi_{p n_{0}}\left(\bar{g}_{i}\right)=\pi_{p n_{0}}\left(\bar{h}_{i}\right)$ for every $0 \leq i \leq n$. The $\operatorname{arcs} G$ and $H$ are said to have mirrored $p$-shape if $n=m$ and $\pi_{p n_{0}}\left(\bar{g}_{i}\right)=\pi_{p n_{0}}\left(\bar{h}_{n-i}\right)$ for every $0 \leq i \leq n$.

Example 1.5. In Figure 4, the arc $H$ is $p$-symmetric. The arc between $\bar{u}$ and $\bar{w}$ has the same mirrored $p$-shape as the arc between $\bar{w}$ and $\bar{v}$.

Definition 22. Let $\bar{w}, \bar{v}, \bar{u} \in \mathcal{C}$. Choose $k \in \mathbb{N}$ and $v \in B_{f}$ such that $\bar{w}, \bar{v}, \bar{u} \in \mathcal{A}_{v}^{k}$. The point $\bar{w}$ is said to be closer than $\bar{u}$ to $\bar{v}$ if $\left|\pi_{k}(\bar{w})-\pi_{k}(\bar{v})\right|<$ $\left|\pi_{k}(\bar{u})-\pi_{k}(\bar{v})\right|$. 
As the backward itineraries of $\bar{w}, \bar{v}$, and $\bar{u}$ are eventually identical, $k$ and $v$ must exist. As $f \mid \mathbb{I}_{R}$ and $f \mid \mathbb{I}_{L}$ are linear, Definition 22 is independent of $k$.

Using this terminology, we can make some observation about how $\mathcal{C}$ passes through the links of $\mathcal{L}_{p n_{0}, m}$. Let $\bar{w}$ be a $p$-wrapping point with $L_{p}(\bar{w})$ $>0$ and $H$ the largest $p$-symmetry that has $\bar{w}$ for a center. Exactly one end point of $H$ is a $p$-wrapping point of higher $p$-level than $\bar{w}$, all other $p$-wrapping points contained in $H$ have a $p$-level less than $\bar{w}$ (Proposition $23)$. The other end point of $H$, say $\bar{u}$, is a $(p-1)$-wrapping point and the difference of the $(p-1)$-level of $\bar{w}$ and the $(p-1)$-level of $\bar{u}$ is less than $n_{0}$ (Proposition 24). Note that $\bar{u}$ may also be a $p$-wrapping point as $\widetilde{\mathrm{W}}_{p} \subset \widetilde{\mathrm{W}}_{p-1}$.

Proposition 23 [15, Proposition 34]. Let $p \in \mathbb{N}$ and $\bar{w} \in \widetilde{\mathrm{W}}_{p} \backslash\{\bar{c}\}$ be such that $L_{p}(\bar{w}) \neq 0$. Let $H$ be the union of all p-symmetries with center $\bar{w}$. There exists $\bar{v}$ such that $\bar{v}$ is the closest element of $\widetilde{\mathrm{W}}_{p}$ to $\bar{w}$ with $L_{p}(\bar{v})>L_{p}(\bar{w})$. Furthermore, $\bar{v}$ is an end point of $H$.

Proposition 24 [15, Proposition 36]. Let $p \in \mathbb{N}, \bar{w} \in \widetilde{\mathrm{W}}_{p} \backslash\{c\}$ and $H$ the union of all p-symmetries with $\bar{w}$ as the center. Let $\bar{v}$ be the end point of $H$ with $L_{p}(\bar{v})>L_{p}(\bar{w}), \bar{u}$ the other end point of $H$, and $c_{i}=\pi_{p n_{0}}(\bar{v})$ where $0 \leq i<n_{0}$. If $L_{p}(\bar{w})>i$, then $\bar{u} \in \widetilde{\mathrm{W}}_{p}$ and $0<L_{p}(\bar{w})-L_{p}(\bar{u})<n_{0}$.

EXAMPLE 1.6. Let $\Upsilon$ be the tent map with kneading sequence $R L R R C$. Figure 4 shows the $p$-wrapping points and the $p$-levels of the $p$-wrapping points and an example of $\bar{w}, H$, and $\bar{v}$ from Proposition 23. Also included is $\bar{u}$ from Proposition 24 .

TheOREM 25 [15, Theorems 54 and 55]. Let $p \leq r \in \mathbb{N}$. Let $\bar{w} \in \widetilde{\mathrm{W}}_{r} \cap \widetilde{\Phi}_{r, i}$ where $0 \leq i<n_{0}$ is such that $\bar{w}$ is not the first element in $\mathrm{W}_{r} \cap \widetilde{\Phi}_{r, i}$. Let $\bar{u}$ be the closest $r$-wrapping point to $\bar{w}$ such that $\bar{u} \in \widetilde{\Phi}_{r, i}$ and $\bar{u} \triangleleft \bar{w}$, and $\bar{v}$ the closest $r$-wrapping point to $\bar{w}$ such that $\bar{v} \in \widetilde{\Phi}_{r, i}$ and $\bar{w} \triangleleft \bar{v}$. Let $G$ be the arc that lies between $\bar{v}$ and $\bar{w}$, and $H$ the arc that lies between $\bar{w}$ and $\bar{u}$. Then:

(i) The arcs $G$ and $H$ are $r$-symmetric.

(ii) If $G$ and $H$ have the same $p$-shape, then $\bar{w} \in \widetilde{\mathrm{W}}_{r+1}$.

(iii) If $\bar{w} \in \widetilde{\mathrm{W}}_{r+1}$, then $G \cup H$ is an r-symmetry.

EXAMPLE 1.7. Consider Figure 5 . The points $\bar{a}, \bar{b}, \bar{e}$, and $\bar{d}$ are elements of $\mathrm{w}_{p} \cap \widetilde{\Phi}_{p 5,1}$. First let $\bar{b}$ be the $\bar{w}$ in Theorem 25 . Then $\bar{a}=\bar{u}$ and $\bar{e}=\bar{v}$. The arc between $\bar{a}$ and $\bar{b}$ and the arc between $\bar{b}$ and $\bar{e}$ are $p$-symmetric. However, as the arc between $\bar{a}$ and $\bar{b}$ does not have the same $p$-shape as the $\operatorname{arc}$ between $\bar{b}$ and $\bar{e}$, i.e., the arc between $\bar{a}$ and $\bar{e}$ is not $p$-symmetric, it follows that $\bar{b} \notin \widetilde{\mathrm{W}}_{p+1}$.

Now let $\bar{e}$ be the $\bar{w}$ from Theorem 25 . Then $\bar{b}=\bar{u}$ and $\bar{d}=\bar{v}$. The arc between $\bar{b}$ and $\bar{e}$ and the arc between $\bar{e}$ and $\bar{d}$ are $p$-symmetric. As the arc between $\bar{b}$ and $\bar{e}$ has the same $p$-shape as the arc between $\bar{e}$ and $\bar{d}$, we have 


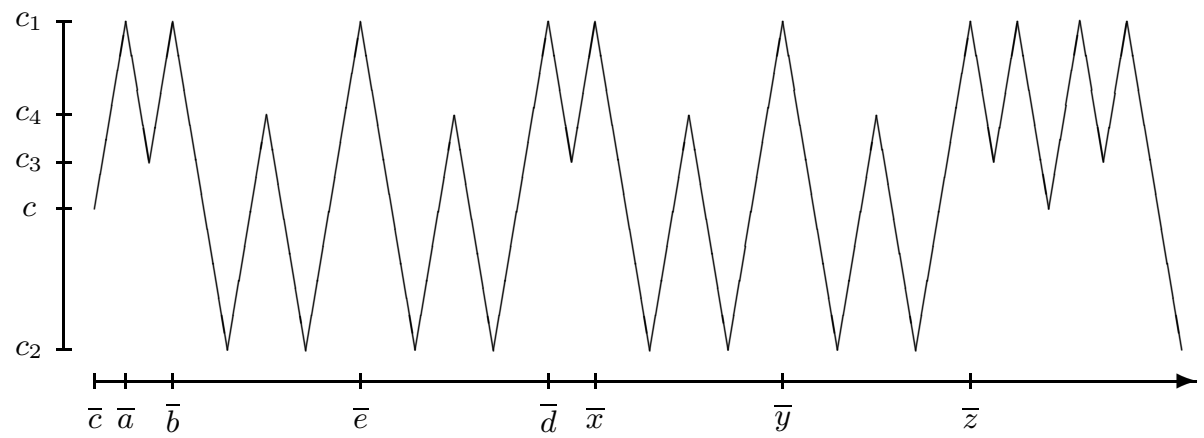

Fig. 5. $\pi_{p 5}: \mathcal{C} \rightarrow \mathbb{I}$ and selected $p$-wrapping points from Examples 1.7 and 1.8

$\bar{e} \in \widetilde{\mathrm{W}}_{p+1}$, and hence the arc between $\bar{b}$ and $\bar{d}$ is $p$-symmetric. In Definition 26 , we define the arc between $\bar{b}$ and $\bar{d}$ as the $(p+1)$-forced symmetry of $\bar{e}$.

Definition 26. Let $r \in \mathbb{N}, \bar{w} \in \widetilde{\mathrm{W}}_{r+1} \backslash\{\bar{c}\}$ and $\pi_{r n_{0}}(\bar{w})=c_{i}$ for some $0 \leq i<n_{0}$. Let $\bar{u}$ be the closest $r$-wrapping point to $\bar{w}$ such that $\bar{u} \in \widetilde{\Phi}_{r, i}$ and $\bar{u} \triangleleft \bar{w}$, and $\bar{v}$ the closest $r$-wrapping point to $\bar{w}$ such that $\bar{v} \in \widetilde{\Phi}_{r, i}$ and $\bar{w} \triangleleft \bar{v}$. Let $H$ be the arc that lies between $\bar{u}$ and $\bar{v}$. The $\operatorname{arc} H$ is the $(r+1)$-forced symmetry of $\bar{w}$.

TheOREM 27 [15, Theorems 58 and 59]. Let $p \leq r \in \mathbb{N}, \bar{w} \in \widetilde{\Phi}_{r+1, i} \cap \widetilde{\mathrm{W}}_{r+1}$ for some $0 \leq i<n_{0}, \bar{w} \neq \bar{c}$, and $H$ the $(r+1)$-forced symmetry of $\bar{w}$. Let $J \subset \mathcal{C}$ be an arc such that $H$ and $J$ have the same p-shape. Then $\bar{\kappa}_{J} \in \widetilde{\mathrm{W}}_{r+1}$.

Example 1.8. Consider Figure 5. The point $\bar{e} \in \mathrm{W}_{p+1}$ and the arc that lies between $\bar{b}$ and $\bar{d}$ is the $(p+1)$-forced symmetry of $\bar{e}$. The arc that lies between $\bar{x}$ and $\bar{z}$ has the same $p$-shape as the arc between $\bar{b}$ and $\bar{d}$, hence $\bar{y} \in \widetilde{\mathrm{W}}_{p+1}$.

Using Theorems 25 and 27 , we can determine the $p$-level of a $p$-wrapping point by looking at the $p$-pseudosymmetries which have the desired $p$-wrapping point as the center. Hence we are interested in what properties the images of these forced symmetries have under $\bar{h}$. Thus we extend our "fudged" map, $\bar{h}_{q, p}:\left(\widetilde{\Phi}_{q}\right)^{\prime} \rightarrow \widetilde{\Phi}_{p}$, to the map $\widetilde{h}_{q, p}$ which takes arcs of $\mathcal{C}^{\prime}$ whose end points are $q$-special points to arcs of $\mathcal{C}$ whose end points are $p$-special points.

Definition 28. Fix $m, p, q, r \in \mathbb{N}$ such that $\bar{h}\left(\mathcal{L}_{q, r}^{\prime}\right) \prec \mathcal{L}_{p, m}$. Define

- $\Lambda_{p} \equiv\left\{D \subset \mathcal{C} \mid D\right.$ is an arc and $\left.\partial D \subset \widetilde{\Phi}_{p}\right\}$,

- $\Lambda_{q}^{\prime} \equiv\left\{D^{\prime} \subset \mathcal{C}^{\prime} \mid D^{\prime}\right.$ is an arc and $\left.\partial D^{\prime} \subset \widetilde{\Phi}_{q}^{\prime}\right\}$,

- $\widetilde{h}_{q, p}: \Lambda_{q}^{\prime} \rightarrow \Lambda_{p}$ to be the function that takes $D^{\prime} \in \Lambda_{q}^{\prime}$ with end points $\bar{x}^{\prime}$ and $\bar{y}^{\prime}$ to the arc of $\mathcal{C}$ that lies between $\bar{h}_{q, p}\left(\bar{x}^{\prime}\right)$ and $\bar{h}_{q, p}\left(\bar{y}^{\prime}\right)$.

Proposition 29 [15, Proposition 65]. Fix m, p, q, r $\in \mathbb{N}$ such that $\bar{h}\left(\mathcal{L}_{q, r}^{\prime}\right)$ $\prec \mathcal{L}_{p, m}$. Let $G^{\prime}, H^{\prime} \in \Lambda_{q}^{\prime}$ be such that $G^{\prime}$ and $H^{\prime}$ have the same $q$-shape (resp. 
mirrored q-shape). Then $\widetilde{h}_{q, p}\left(G^{\prime}\right)$ and $\widetilde{h}_{q, p}\left(H^{\prime}\right)$ have the same $p$-shape (resp. mirrored p-shape).

Proposition 29 tells us that if $\bar{x}^{\prime}, \bar{v}^{\prime} \in \widetilde{\Phi}_{r, i}^{\prime}$ where $r \geq q+1$ and $0 \leq$ $i<n_{0}$, the $r$-forced symmetries of $\bar{v}$ and $\bar{x}$ will map under $\widetilde{h}_{q, p}$ to $p$ pseudosymmetries of the same $p$-shape. These $p$-pseudosymmetries force a certain $p$-level on their centers, $\bar{h}_{q, p}(\bar{v})$ and $\bar{h}_{q, p}(\bar{x})$ (Theorem 27). If we choose $p, m, u, v \in \mathbb{N}$ such that $\mathcal{L}_{p n_{0}, m} \prec \bar{h}\left(\mathcal{L}_{u n_{0}, v}^{\prime}\right.$ ) (possible by Lemma 11(iii)), the $u$-level of the images of $s$-special points, where $s \geq p+1$, are similarly forced. Using these results together, given any $r \geq q+1$ and $0 \leq i, j<n_{0}$ such that $\bar{h}\left(\bar{c}_{i}^{\prime}\right)=\bar{c}_{j}$, we can find $t \in \mathbb{N}$ such that $\widetilde{\Phi}_{t+1, j} \subset \bar{h}_{q, p}\left(\widetilde{\Phi}_{r, i}\right) \subset \widetilde{\Phi}_{t, j}$. Unfortunately, we cannot guarantee that the same $t$ will work for all $i$, thus we restrict our attention to $i=0$, i.e. those special points that project to $c$.

Theorem 30 [15, Theorem 70]. Fix $p, m, q, r, u, v \in \mathbb{N}$ such that $\bar{h}\left(\mathcal{L}_{q n_{0}, r}^{\prime}\right)$ $\prec \mathcal{L}_{p n_{0}, m} \prec \bar{h}\left(\mathcal{L}_{u n_{0}, v}^{\prime}\right)$. There exists $t$ such that

$$
\widetilde{\Phi}_{t, 0} \supset \bar{h}_{q, p}\left(\widetilde{\Phi}_{q+1,0}^{\prime}\right) \supsetneq \widetilde{\Phi}_{t+1,0} \supset \bar{h}_{q, p}\left(\widetilde{\Phi}_{q+2,0}^{\prime}\right) \supsetneq \widetilde{\Phi}_{t+2,0} \supset \ldots
$$

As Theorem 30 applies only to those special points that project to $c$, we pay special attention to the arcs that lie between these special points.

Definition 31. Fix $p \in \mathbb{N}$. As $\widetilde{\Phi}_{p, 0} \subset \widetilde{\mathrm{W}}_{p}$ and $\widetilde{\mathrm{W}}_{p}$ is countable, $\widetilde{\Phi}_{p, 0}$ is countable. Thus $\widetilde{\Phi}_{p, 0}$ partitions $\mathcal{C}$ into countably many arcs. Denote this partition by $\mathcal{P}_{p}$. We call $H \subset \mathcal{C}$ a $p$-gap of $\mathcal{C}$ provided $H \in \mathcal{P}_{p}$. Observe that for each $p$-gap, $H, c \notin \pi_{p n_{0}}(\stackrel{\circ}{H})$, and $\pi_{p n_{0}}(\partial H)=\{c\}$.

EXAMPLE 1.9. Let $\Upsilon$ be the tent map with kneading sequence $R L R R C$. Figure 6 shows the $p$-gaps of $\mathcal{C}$. Although the $p$-gaps of $\mathcal{C}$ are subsets of $\mathcal{C}$, it is often helpful to think of $p$-gaps in terms of the graph of the map $\pi_{p n_{0}}$.

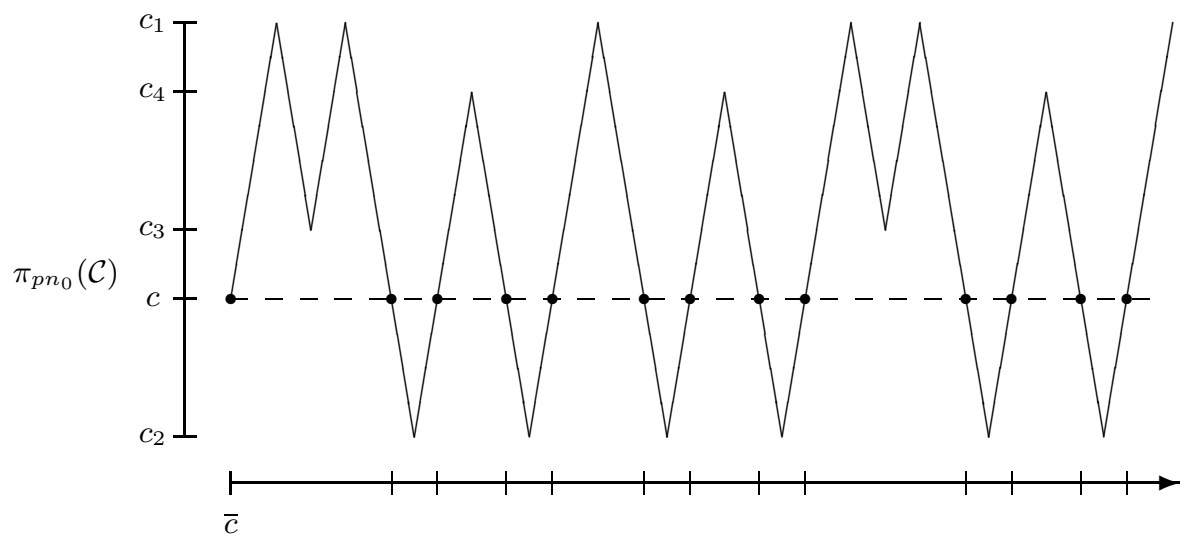

Fig. 6. The $p$-gaps of $\mathcal{C}$ and the set $\pi_{p n_{0}}\left(\widetilde{\Phi}_{p, 0}\right)(\bullet)$ 
Note that the intersection of two distinct $p$-gaps is either a $p$-special point or the empty set. We can now restate Theorem 30 using gaps.

Theorem 32 (Theorem 30 restated). Fix $p, m, q, r, u, v \in \mathbb{N}$ such that $\bar{h}\left(\mathcal{L}_{q n_{0}, r}^{\prime}\right) \prec \mathcal{L}_{p n_{0}, m} \prec \bar{h}\left(\mathcal{L}_{u n_{0}, v}^{\prime}\right)$. There exists $t$ such that for any $k \in \mathbb{N}_{+}$, if $G^{\prime}$ is a $(q+k)$-gap of $\mathcal{C}^{\prime}$, then $\widetilde{h}_{q, p}\left(G^{\prime}\right)$ is a group of $(t+k-1)$-gaps of $\mathcal{C}$ and is contained in one $(t+k)$-gap of $\mathcal{C}$.

Proposition 33 [15, Proposition 35]. Fix $p \in \mathbb{N}$. If $G$ is a p-gap of $\mathcal{C}$, then $G$ is p-symmetric.

Definition 34. Fix $p \in \mathbb{N}$. Let $G$ and $H$ be distinct $p$-gaps of $\mathcal{C}$. Define $G$ to be less than $H$ in the $\mathcal{C}$-induced order, denoted $G \triangleleft H$, if for every $\bar{x} \in G$ and every $\bar{y} \in H$, either $\bar{x} \triangleleft \bar{y}$ or $\bar{x}=\bar{y}$.

Definition 35. Fix $p, q \in \mathbb{N}$. Let $G$ be a $p$-gap of $\mathcal{C}$ with $G \cap \mathrm{W}_{p}=$ $\left\{\bar{g}_{0}, \bar{g}_{1}, \ldots, \bar{g}_{n}\right\}$, and $H$ a $q$-gap of $\mathcal{C}$ with $H \cap \mathrm{W}_{q}=\left\{\bar{h}_{0}, \bar{h}_{1}, \ldots, \bar{h}_{m}\right\}$. The gaps, $G$ and $H$, are said to be the same type if $n=m$ and $\pi_{p n_{0}}\left(\bar{g}_{i}\right)=\pi_{q n_{0}}\left(\bar{h}_{i}\right)$ for all $0 \leq i \leq n$.

EXAMPLE 1.10. Let $\Upsilon$ be the tent map with kneading sequence $R L R R C$. The first and ninth $p$-gaps of $\mathcal{C}$ are the same type. See Figure 6 . The second and fourth $p$-gaps are also the same type, but are a different type than the first $p$-gap.

Definition 36. Fix $p \in \mathbb{N}$. Let $G$ be a $p$-gap of $\mathcal{C}$. Define

$T(G)=\min \left\{L_{q}\left(\bar{\kappa}_{H}\right) \mid q \in \mathbb{N}\right.$ and $H$ is a $q$-gap of the same type as $\left.G\right\}$.

EXAMPLE 1.11. Let $\Upsilon$ be the tent map with kneading sequence $R L R R C$. By looking at Figure 4, we can see that the level of the first $p$-gap of $\mathcal{C}$ is 3. The level of the second $p$-gap is 2 . The level of the ninth $p$-gap is 3 , because the ninth $p$-gap is the same type as the first $p$-gap.

Proposition 37 [15, Corollary 42]. Fix $p, q \in \mathbb{N}$. Let $G$ be a $p$-gap of $\mathcal{C}$ and $H$ a q-gap of $\mathcal{C}$. The gaps $G$ and $H$ are the same type if and only if $T(G)=T(H)$.

Definition 38. Fix $p \in \mathbb{N}$. Define $F_{p}$ to be the first $p$-gap of $\mathcal{C}$ in $\mathcal{C}$ induced order, and $\varphi=T\left(F_{p}\right)$.

Note that $\varphi$ does not depend on $p$, and $\varphi<n_{0}$. Throughout the rest of this paper $\varphi$ denotes this fixed constant.

Corollary 39 (of Theorem 30). Fix $p, m, q, r, u, v \in \mathbb{N}$ with $\bar{h}\left(\mathcal{L}_{q n_{0}, r}^{\prime}\right)$ $\prec \mathcal{L}_{p n_{0}, m} \prec \bar{h}\left(\mathcal{L}_{u n_{0}, v}^{\prime}\right)$. If $\widetilde{h}_{q, p}\left(F_{q+1}^{\prime}\right)=F_{t}$ for some $t \in \mathbb{N}$, then for every $k \in \mathbb{N}_{+}, \bar{h}_{q, p}\left(\widetilde{\Phi}_{q+k, 0}^{\prime}\right)=\widetilde{\Phi}_{t+k-1,0}$. Alternatively stated, if $\widetilde{h}_{q, p}\left(F_{q+1}^{\prime}\right)=F_{t}$ for some $t \in \mathbb{N}$, then for every $k \in \mathbb{N}_{+}$, any $(q+k)$-gap of $\mathcal{C}^{\prime}$ will map under $\widetilde{h}_{q, p}$ to exactly one $(t+k-1)$-gap of $\mathcal{C}$. 
Remark. Informally, Corollary 39 says that if the first gap of $\mathcal{C}^{\prime}$ maps onto the first gap of $\mathcal{C}$, then gaps go to gaps.

Definition 40. Fix $p, m, q, r, u, v \in \mathbb{N}$ such that $\bar{h}\left(\mathcal{L}_{q n_{0}, r}^{\prime}\right) \prec \mathcal{L}_{p n_{0}, m} \prec$ $\bar{h}\left(\mathcal{L}_{u n_{0}, v}^{\prime}\right)$. Let $t$ be the integer from Theorem 30 and $k \in \mathbb{N}_{+}$. Let $H^{\prime}$ be a $(q+k)$-gap of $\mathcal{C}^{\prime}$. The $(q+k)$-structure of $H^{\prime}$ is the set

$$
\overrightarrow{\mathcal{S}}_{q+k}\left(H^{\prime}\right) \equiv\left\{G \subset \mathcal{C} \mid G \text { is a }(t+k-1) \text {-gap and } G \subset \widetilde{h}_{q, p}\left(H^{\prime}\right)\right\}
$$

in $\mathcal{C}$-induced order.

Let $H$ be a $(t+k)$-gap of $\mathcal{C}$. The $(t+k)^{\prime}$-structure of $H$ is the set

$$
\overrightarrow{\mathcal{S}}_{t+k}^{\prime}(H) \equiv\left\{G^{\prime} \subset \mathcal{C}^{\prime} \mid G^{\prime} \text { is a }(q+k) \text {-gap and } G^{\prime} \subset \widetilde{h}_{p, u}^{\prime}(H)\right\}
$$

in $\mathcal{C}^{\prime}$-induced order.

Definition 41. Fix $q, p \in \mathbb{N}$. Let $\mathcal{H}=\left\{H_{0}, H_{1}, \ldots, H_{n}\right\}$ be an ordered set of $q$-gaps and $\mathcal{G}=\left\{G_{0}, G_{1}, \ldots, G_{m}\right\}$ an ordered set of $p$-gaps. The set $\mathcal{H}$ is defined to be similar to the set $\mathcal{G}$, denoted $\mathcal{H} \sim \mathcal{G}$, if $n=m$ and $T\left(H_{i}\right)=T\left(G_{i}\right)$ for all $0 \leq i \leq n$.

Proposition 42 [15, Corollary 71]. Fix $p, m, q, r, u, v \in \mathbb{N}$ such that $\bar{h}\left(\mathcal{L}_{q n_{0}, r}^{\prime}\right) \prec \mathcal{L}_{p n_{0}, m} \prec \bar{h}\left(\mathcal{L}_{u n_{0}, v}^{\prime}\right)$. Let $s, b>q \in \mathbb{N}, G^{\prime}$ an s-gap and $H^{\prime} a$ b-gap such that $T\left(G^{\prime}\right)=T\left(H^{\prime}\right)$. Then $\overrightarrow{\mathcal{S}}_{b}\left(H^{\prime}\right) \sim \overrightarrow{\mathcal{S}}_{s}\left(G^{\prime}\right)$.

Proposition 43 [15, Proposition 74]. Fix $p, m, q, r, u, v \in \mathbb{N}$ such that $\bar{h}\left(\mathcal{L}_{q n_{0}, r}^{\prime}\right) \prec \mathcal{L}_{p n_{0}, m} \prec \bar{h}\left(\mathcal{L}_{u n_{0}, v}^{\prime}\right)$. Let $t$ be the integer from Theorem 30 . Let $s, b>t \in \mathbb{N}, G$ an $s$-gap and $H$ a b-gap such that $T(G)=T(H)$. Then $\overrightarrow{\mathcal{S}_{b}^{\prime}}(H) \sim \overrightarrow{\mathcal{S}}_{s}^{\prime}(G)$.

Definition 44. Fix $p \in \mathbb{N}$ and let $G$ be a $p$-gap. Define the orientation of $G$ as follows: $G$ is an up gap if $\pi_{p n_{0}}(G) \subset \mathbb{I}_{R}$, and $G$ is a down gap if $\pi_{p n_{0}}(G) \subset \mathbb{I}_{L}$. Additionally, $G$ is an even gap if it has the same orientation as $F_{p}$, and $G$ is an odd gap if it has the opposite orientation of $F_{p}$.

Similarly, if $\bar{w} \in \widetilde{\mathrm{W}}_{p} \cup\left\{\bar{c}_{j} \mid 0<j<n_{0}\right\}$ and $\pi_{p n_{0}}(\bar{w}) \neq c$, then $\bar{w}$ is an up point if $\pi_{p n_{0}}(\bar{w}) \in \mathbb{I}_{R}$, a down point if $\pi_{p n_{0}}(\bar{w}) \in \mathbb{I}_{L}$, an even point if $\bar{\kappa}_{F_{p}}$ has the same orientation, and an odd point if $\bar{\kappa}_{F_{p}}$ has the opposite orientation.

Note that two adjacent $p$-gaps of $\mathcal{C}$ have opposite orientation unless their point of intersection is a $(p+1)$-wrapping point, in which case both $p$-gaps are even and type $\varphi$.

Lemma 45 [15, Lemmas 81 and 82]. Fix $p, m, q, r, u, v \in \mathbb{N}$ such that $\bar{h}\left(\mathcal{L}_{q n_{0}, r}^{\prime}\right) \prec \mathcal{L}_{p n_{0}, m} \prec \bar{h}\left(\mathcal{L}_{u n_{0}, v}^{\prime}\right)$. Let $t$ be the integer from Theorem 58 . Let $k \in \mathbb{N}_{+}$and $G^{\prime} a(q+k)$-gap of $\mathcal{C}^{\prime}$. Then $\widetilde{h}_{q, p}\left(\bar{\kappa}_{G^{\prime}}\right)$ is an odd $(t+k-1)$ wrapping point if and only if $\bar{\kappa}_{G^{\prime}}$ is an odd $(q+k)$-wrapping point. 
4. Main result. In this section we prove our main result. We begin by showing that for appropriate choice of $t$ (depending on $p$ and $q$ ) and any $k \in \mathbb{N}_{+}$, the image of any $(q+k)$-gap of $\mathcal{C}^{\prime}$ under $\widetilde{h}_{q, p}$ is exactly one $(t+k-1)$-gap of $\mathcal{C}$ (Theorem 58). Informally, gaps go to gaps. We then prove our main result (Theorem 63) by showing that when gaps go to gaps, the kneading sequences of the bonding maps must be identical. The lemmas of this section are technical results needed to prove the two main theorems.

Lemma 46 establishes the existence and non-existence of types of $p$-gaps of $\mathcal{C}$ based on the kneading sequence of $f$.

Lemma 46. Fix $p, n \in \mathbb{N}, n \bmod n_{0} \neq 0$, and let $k$ be the smallest odd integer such that $f^{k}(c) \in \mathbb{I}_{L}$. Note that $k \leq n_{0}$.

(i) There exists a p-gap of $\mathcal{C}$ whose center has a p-level of $n$ if and only if $c \in f^{n}\left(\mathbb{I}_{L}\right)$. of $n$.

(ii) If $n$ is even, then there exists a p-gap of $\mathcal{C}$ whose center has a p-level

(iii) If $n$ is odd, then there exists a p-gap of $\mathcal{C}$ whose center has a p-level of $n$ if and only if $n \geq k-2$.

Proof. (i) $\Rightarrow$ Suppose that $H$ is a $p$-gap of $\mathcal{C}$ with $L_{p}\left(\bar{\kappa}_{H}\right)=n$. As $\pi_{p n_{0}+n} \mid H$ is a homeomorphism (Proposition 33), and $\pi_{p n_{0}+n}\left(\bar{\kappa}_{H}\right)=c$, we have $\pi_{p n_{0}+n}(\partial H) \cap \mathbb{I}_{L} \neq \emptyset$. Therefore, $c \in f^{n}\left(\mathbb{I}_{L}\right)$.

$\Leftarrow$ Suppose $c \in f^{n}\left(\mathbb{I}_{L}\right)$. Let $x=\max \left\{y \in \mathbb{I}_{L} \mid f^{n}(y)=c\right\}$, and note that $x \neq c$ as $n \bmod n_{0} \neq 0$. Let $J$ be an $\operatorname{arc}$ of $\mathcal{C}$ such that $\pi_{p n_{0}+n} \mid J$ is a homeomorphism and $\pi_{p n_{0}+n}(J)=\mathbb{I}$. Then $\pi_{p n_{0}+n}^{-1}([x, 1-x]) \cap J$ is a $p$-gap of $\mathcal{C}$ such that the $p$-level of its center is $n$.

(ii) If $c_{3} \in \mathbb{I}_{L}$, then $c \in f\left(\mathbb{I}_{L}\right)=\left[c_{3}, c_{1}\right]$. Hence $\mathbb{I} \subset f^{2}\left(\mathbb{I}_{L}\right)$, and therefore $\mathbb{I} \subset f^{i}\left(\mathbb{I}_{L}\right)$ for all $i \geq 2$. The result follows from (i).

Assume that $c_{3} \in \mathbb{I}_{R}$. From kneading theory, $c_{4} \in \mathbb{I}_{R}$, and hence $\mathbb{I}_{L} \subset$ $f^{2}\left(\mathbb{I}_{L}\right)=\left[c_{2}, c_{4}\right]$. Thus $\mathbb{I}_{L} \subset f^{n-2}\left(\mathbb{I}_{L}\right) \subset f^{n}\left(\mathbb{I}_{L}\right)$ for all even $n \in \mathbb{N}_{+}$. The result follows from (i).

(iii) For all odd $i, c_{1} \in f^{i}\left(\mathbb{I}_{L}\right)$ as $c \in f^{i-1}\left(\mathbb{I}_{L}\right)$ ((ii) and (i)). Thus for all odd $i \leq k, f^{i}\left(\mathbb{I}_{L}\right)=\left[c_{2+i}, c_{1}\right]$. Hence $c \notin f^{i}\left(\mathbb{I}_{L}\right)$ for all odd $i<k-2$, and $c \in f^{i}\left(\mathbb{I}_{L}\right)$ for all odd $i \geq k-2$. The result follows by (i).

Definition 47. Fix $p \in \mathbb{N}$. Let $G$ be a $p$-gap of $\mathcal{C}$ and $G \cap \mathrm{w}_{p}=$ $\left\{\bar{g}_{0}, \bar{g}_{1}, \ldots, \bar{g}_{n}\right\}$. For each $0 \leq i<n$, let $L_{i}$ be the arc that lies between $\bar{g}_{i}$ and $\bar{g}_{i+1}$. Each $L_{i}$ is a leg of $G$, and $L_{0}$ is the first leg of $G$.

Lemma 48 [15, Lemma 44]. Fix $p \in \mathbb{N}$. Let $G$ be a p-gap of $\mathcal{C}$ with first leg L. Then $\pi_{p n_{0}}(G)=\pi_{p n_{0}}(L)$.

Lemma 49 predicts the $(q+k)$-structure of $(q+k)$-gaps based on the $(q+k)$-structure of "shorter" $(q+k)$-gaps. Lemma 50 does the same thing for the $(t+k)^{\prime}$-structure of $(t+k)$-gaps. 
Lemma 49. Fix $p, m, q, r, u, v \in \mathbb{N}$ with $\bar{h}\left(\mathcal{L}_{q n_{0}, r}^{\prime}\right) \prec \mathcal{L}_{p n_{0}, m} \prec \bar{h}\left(\mathcal{L}_{u n_{0}, v}^{\prime}\right)$. Let $t$ be the integer from Theorem 30 . Let $k \in \mathbb{N}_{+}$and $G^{\prime}$ be $a(q+k)$-gap of $\mathcal{C}^{\prime}$ such that $\overrightarrow{\mathcal{S}}_{q+k}\left(G^{\prime}\right)$ is more than one $(t+k-1)$-gap of $\mathcal{C}$. Set $\pi_{(q+k) n_{0}}\left(G^{\prime}\right)=$ $\left[c^{\prime}, x^{\prime}\right]$. Let $H^{\prime}$ be a $(q+k)$-gap of $\mathcal{C}^{\prime}$. If $\pi_{(q+k) n_{0}}\left(H^{\prime}\right) \not \subset\left(1-x^{\prime}, x^{\prime}\right)$ then $\overrightarrow{\mathcal{S}}_{q+k}\left(H^{\prime}\right)$ is more than one $(t+k-1)$-gap.

Proof. Let $L^{\prime}$ be the first leg of $G^{\prime}$ and $K^{\prime}$ the first leg of $H^{\prime}$. By Lemma $48, \pi_{(q+k) n_{0}}\left(L^{\prime}\right)=\pi_{(q+k) n_{0}}\left(G^{\prime}\right)=\left[c^{\prime}, x^{\prime}\right]$ and $\pi_{(q+k) n_{0}}\left(K^{\prime}\right)=\pi_{(q+k) n_{0}}\left(H^{\prime}\right)$. By [15, proof of Proposition 79], $\widetilde{h}_{q, p}\left(L^{\prime}\right)$ contains the first $(t+k-1)$-gap of $\overrightarrow{\mathcal{S}}_{q+k}\left(G^{\prime}\right)$ and the $(t+k-1)$-forced symmetry of the last end point of the first gap of $\overrightarrow{\mathcal{S}}_{q+k}\left(G^{\prime}\right)$. Suppose $\pi_{(q+k) n_{0}}\left(H^{\prime}\right) \not \subset\left(1-x^{\prime}, x^{\prime}\right)$. Then $\pi_{(q+k) n_{0}}\left(K^{\prime}\right) \not \subset$ $\left(1-x^{\prime}, x^{\prime}\right)$ (Lemma 48). Hence, by the symmetry of $g$, there is an arc, say $M^{\prime}$, such that $M^{\prime} \subset K^{\prime}$ and $M^{\prime}$ and $L^{\prime}$ have the same $q$-shape. Therefore, $\widetilde{h}_{q, p}\left(M^{\prime}\right)$ and $\widetilde{h}_{q, p}\left(L^{\prime}\right)$ have the same $p$-shape (Proposition 29$)$. Thus $\widetilde{h}_{q, p}\left(M^{\prime}\right)$ contains an arc with the same $p$-shape as the $(t+k-1)$-forced symmetry of the last end point of the first $(t+k-1)$-gap of $\overrightarrow{\mathcal{S}}_{q+k}\left(G^{\prime}\right)$. Hence, by Theorem $27, \widetilde{h}_{q, p}\left(M^{\prime}\right)$ contains a $(t+k-1)$-wrapping point which projects to $c$ in its interior. Thus $\widetilde{h}_{q, p}\left(M^{\prime}\right)$ contains a $(t+k-1)$-gap, and therefore $\overrightarrow{\mathcal{S}}_{q+k}\left(H^{\prime}\right)$ is more than one $(t+k-1)$-gap.

Lemma 50. Fix $p, m, q, r, u, v \in \mathbb{N}$ with $\bar{h}\left(\mathcal{L}_{q n_{0}, r}^{\prime}\right) \prec \mathcal{L}_{p n_{0}, m} \prec \bar{h}\left(\mathcal{L}_{u n_{0}, v}^{\prime}\right)$. Let $t$ be the integer from Theorem 30 . Let $k \in \mathbb{N}_{+}$and $G$ be a $(t+k)$-gap of $\mathcal{C}$ such that $\overrightarrow{\mathcal{S}}_{t+k}^{\prime}(G)$ is more than one $(q+k)$-gap of $\mathcal{C}^{\prime}$. Set $\pi_{(t+k) n_{0}}(G)=[c, x]$. Let $H$ be a $(t+k)$-gap of $\mathcal{C}$. If $\pi_{(t+k) n_{0}}(H) \not \subset(1-x, x)$ then $\overrightarrow{\mathcal{S}}_{t+k}^{\prime}(H)$ is more than one $(q+k)$-gap.

Proof. Similar to the proof of Lemma 49.

Definition 51. Let $\left[c, c_{j}\right]=\pi_{0}\left(F_{0}\right)$ and $\left[c^{\prime}, c_{i}^{\prime}\right]=\pi_{0}\left(F_{0}^{\prime}\right)$. Define $\mathcal{I}=$ $\left[1-c_{j}, c_{j}\right]$ and $\mathcal{I}^{\prime}=\left[1-c_{i}^{\prime}, c_{i}^{\prime}\right]$.

Lemmas 52 and 53 identify properties of the image of the first gap of $\mathcal{C}^{\prime}$ under $\widetilde{h}_{q, p}$ and the image of the first gap of $\mathcal{C}$ under $\widetilde{h}_{p, u}^{\prime}$ respectively.

Lemma 52. Fix $p, m, q, r, u, v \in \mathbb{N}$ with $\bar{h}\left(\mathcal{L}_{q n_{0}, r}^{\prime}\right) \prec \mathcal{L}_{p n_{0}, m} \prec \bar{h}\left(\mathcal{L}_{u n_{0}, v}^{\prime}\right)$. Let $t$ be the integer from Theorem 30 . If $k \in \mathbb{N}_{+}$, then $\pi_{(t+k-1) n_{0}}\left(\widetilde{h}_{q, p}\left(F_{q+k}^{\prime}\right)\right)$ $\subsetneq \mathcal{I}$ and $\pi_{(t+k-1) n_{0}}\left(\widetilde{h}_{q, p}\left(F_{q+k}^{\prime}\right)\right) \subsetneq \mathbb{I}$.

Proof. If $\widetilde{h}_{q, p}\left(F_{q+1}^{\prime}\right)=F_{t}$, the result follows. Assume $\widetilde{h}_{q, p}\left(F_{q+1}^{\prime}\right) \neq F_{t}$. As $\overrightarrow{\mathcal{S}}_{q+k}\left(F_{q+k}\right) \sim \overrightarrow{\mathcal{S}}_{q+2}\left(F_{q+2}\right)$ for all $k \geq 1$ (Proposition 42 ), we need only prove the result for $k=2$. Let $G$ be the first $(t+1)$-gap of $\mathcal{C}$ not in $\overrightarrow{\mathcal{S}}_{q+2}\left(F_{q+2}^{\prime}\right)$. As the image of an odd $(q+2)$-gap under $\widetilde{h}_{q, p}$ is either a $(t+1)$-gap or a $(t+2)$-gap [15, Lemma 81], the second $(q+2)$-gap of $\mathcal{C}^{\prime}$ must map to $G$, and 
hence $\widetilde{h}_{p, u}^{\prime}(G)$ is an odd $(q+2)$-gap. Thus $\overrightarrow{\mathcal{S}}_{t+1}^{\prime}(G)$ is more than one $(q+1)$ gap. Furthermore, as the image of an odd $(t+1)$-gap under $\widetilde{h}_{p, u}^{\prime}$ is either a $(q+1)$-gap or a $(q+2)$-gap [15, Lemma 82], the image under $\widetilde{h}_{p, u}^{\prime}$ of any odd $(t+1)$-gap of $\overrightarrow{\mathcal{S}}_{q+2}\left(F_{q+2}\right)$ is one odd $(q+1)$-gap. Therefore, by Lemma 50 , if $H \in \overrightarrow{\mathcal{S}}_{q+2}\left(F_{q+2}\right)$ is an odd gap, then $\pi_{(t+1) n_{0}}(H) \subsetneq \pi_{(t+1) n_{0}}(G)$ and $\pi_{(t+1) n_{0}}(H) \subsetneq \mathcal{I}$. Hence $\pi_{(t+1) n_{0}}\left(\widetilde{h}_{q, p}\left(F_{q+2}^{\prime}\right)\right) \subsetneq \mathbb{I}$ and if $\pi_{(t+1) n_{0}}\left(F_{q+2}\right) \subset \mathcal{I}$ then $\pi_{(t+1) n_{0}}\left(F_{q+2}\right) \subsetneq \mathcal{I}$.

Suppose that $\pi_{(t+1) n_{0}}\left(\widetilde{h}_{q, p}\left(F_{q+2}^{\prime}\right)\right) \not \subset \mathcal{I}$. Let $L$ be the first leg of $G$ and $\left\{\bar{w}_{0}, \bar{w}_{1}, \ldots, \bar{w}_{d}\right\}=\mathrm{W}_{t+1} \cap\left\{L \cup \widetilde{h}_{q, p}\left(F_{q+2}^{\prime}\right)\right\}$. Let $i=\sup \{k \mid k<d$ and $\left.\pi_{(t+1) n_{0}}\left(\bar{w}_{k}\right) \notin \mathcal{I}\right\}$. Note that $\bar{w}_{i}$ is an even $(t+1)$-wrapping point and $\bar{w}_{i}$ is not in the last $(t+1)$-gap of $\overrightarrow{\mathcal{S}}_{q+2}\left(F_{q+2}\right)$ as that $(t+1)$-gap is type $\boldsymbol{\varphi}$. Let $\bar{w}_{e}$ be the $(t+1)$-wrapping point such that $L_{t+1}\left(\bar{w}_{e}\right) \geq L_{t+1}\left(\bar{w}_{k}\right)$ for all $i<k<d$. Then $L_{t+1}\left(\bar{w}_{e}\right) \geq \varphi$. By Proposition 23, either $\bar{w}_{i}$ or $\bar{w}_{d}$ is contained in a $(t+1)$-symmetry about $\bar{w}_{e}$. However, by the choice of $\bar{w}_{i}$ and $\bar{w}_{d}$ neither can be in a $(t+1)$-symmetry about $\bar{w}_{e}$, a contradiction. Hence $\pi_{(t+1) n_{0}}\left(\widetilde{h}_{q, p}\left(F_{q+2}^{\prime}\right)\right) \subsetneq \mathcal{I}$.

Lemma 53. Fix $p, m, q, r, u, v \in \mathbb{N}$ with $\bar{h}\left(\mathcal{L}_{q n_{0}, r}^{\prime}\right) \prec \mathcal{L}_{p n_{0}, m} \prec \bar{h}\left(\mathcal{L}_{u n_{0}, v}^{\prime}\right)$. Let $t$ be the integer from Theorem 30 and $k \in \mathbb{N}_{+}$. If $\widetilde{h}_{q, p}\left(F_{q+1}^{\prime}\right) \neq F_{t}$, then $\pi_{(q+k) n_{0}}\left(\widetilde{h}_{p, u}^{\prime}\left(F_{t+k}\right)\right) \subsetneq \mathcal{I}^{\prime}$ and $\pi_{(q+k) n_{0}}\left(\widetilde{h}_{p, u}^{\prime}\left(F_{t+k}\right)\right) \subsetneq \mathbb{I}^{\prime}$.

Proof. Similar to the proof of Lemma 52.

In Lemma 54, we look at those $(q+k)$-gaps of $\mathcal{C}^{\prime}$ that map to exactly one $(t+k-1)$-gap of $\mathcal{C}$ and examine the properties that this kind of $(q+k)$-gap has when $\widetilde{h}_{q, p}\left(F_{q+1}^{\prime}\right) \neq F_{t}$. In particular, we show that the $(q+k-1) n_{0}$ projection of this kind of $(q+k)$-gap is not $\mathbb{I}^{\prime}$ and that the center of the $(q+k)$-gap "turns" at an end point that the image of the first $(t+k-1)$ gap of $\mathcal{C}$ under $\widetilde{h}_{p, u}^{\prime}$ "turns" at (see Example 1.12). Lemma 55 is similar to Lemma 54 but looks at gaps of $\mathcal{C}$ instead of gaps of $\mathcal{C}^{\prime}$.

EXAMPLE 1.12. Let $\Upsilon$ be the tent map with kneading sequence $R L R R C$. Figure 7 shows gaps $F_{q+k}^{\prime}$ and $G^{\prime}$. The gap $F_{q+k}^{\prime}$ turns at $\bar{c}_{1}^{\prime}$ and $\bar{c}_{3}^{\prime}$. The gap $G^{\prime}$ only turns at $\bar{c}_{1}^{\prime}$. Note that in Lemma 54 we are not concerned with where $F_{q+k}^{\prime}$ turns, but rather where $\widetilde{h}_{p, u}^{\prime}\left(F_{t+k-1}\right)$ turns.

Lemma 54. Fix $p, m, q, r, u, v \in \mathbb{N}$ with $\bar{h}\left(\mathcal{L}_{q n_{0}, r}^{\prime}\right) \prec \mathcal{L}_{p n_{0}, m} \prec \bar{h}\left(\mathcal{L}_{u n_{0}, v}^{\prime}\right)$. Let $t$ be the integer from Theorem 30 . Let $k \in \mathbb{N}_{+}$and $G^{\prime}$ be $a(q+k)$-gap of $\mathcal{C}^{\prime}$ such that $\widetilde{h}_{q, p}\left(G^{\prime}\right)$ is one $(t+k-1)$-gap. If $\widetilde{h}_{q, p}\left(F_{q+1}^{\prime}\right) \neq F_{t}$, then $\pi_{(q+k-1) n_{0}}\left(G^{\prime}\right) \subsetneq \mathbb{I}^{\prime}$ and $\pi_{(q+k) n_{0}}\left(\bar{\kappa}_{G^{\prime}}\right) \in \pi_{(q+k-1) n_{0}}\left(\widetilde{\mathrm{W}}_{q+k-1}^{\prime} \cap \widetilde{h}_{p, u}^{\prime}\left(F_{t+k-1}\right)\right)$. 


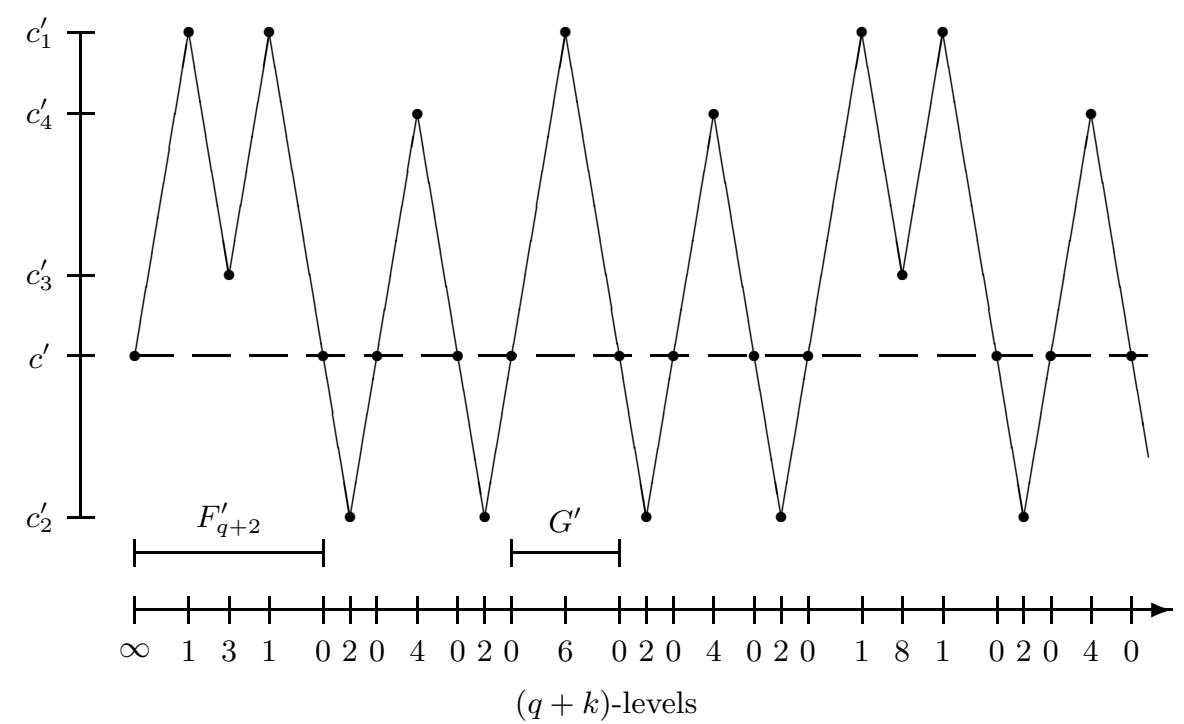

Fig. 7. The $(q+k)$-gaps $F_{q+k}^{\prime}$ and $G^{\prime}$ for the tent map with kneading sequence $R L R R C$

Proof. By Proposition 42 we may assume that $k=3$. Suppose $\widetilde{h}_{q, p}\left(F_{q+1}^{\prime}\right)$ $\neq F_{t}$. Let $D^{\prime}$ be the first $(q+2)$-gap not contained in $\widetilde{h}_{p, u}^{\prime}\left(F_{t+2}\right)$, and $L^{\prime}$ the first leg of $D^{\prime}$. By [15, proof of Proposition 79], $\widetilde{h}_{p, u}^{\prime}\left(F_{t+2}\right) \cup L^{\prime}$ is a subset of the first leg of $F_{q+3}^{\prime}$. Let $[c, x]=\pi_{(q+3) n_{0}}\left(\widetilde{h}_{p, u}^{\prime}\left(F_{t+2}\right) \cup L^{\prime}\right)$. Let $E^{\prime}$ be the first leg of $G^{\prime}$. As $\pi_{(q+3) n_{0}}\left(G^{\prime}\right)=\pi_{(q+3) n_{0}}\left(E^{\prime}\right)$ (Lemma 48), by Propositions 29 and 27, and the symmetry of the map $g^{n_{0}}$, if $\pi_{(q+3) n_{0}}\left(G^{\prime}\right) \not \subset$ $(1-x, x)$ then $\widetilde{h}_{q, p}\left(G^{\prime}\right)$ contains more than one $(t+2)$-gap (see proof of Lemma 49). Hence $\pi_{(q+3) n_{0}}\left(G^{\prime}\right) \subset(1-x, x)$. Therefore, $\pi_{(q+2) n_{0}}\left(G^{\prime}\right) \subset$ $\pi_{(q+2) n_{0}}\left(\widetilde{h}_{p, u}^{\prime}\left(F_{t+2}\right)\right)$, and hence $\pi_{(q+2) n_{0}}\left(G^{\prime}\right) \subsetneq \mathbb{I}^{\prime}$ by Lemma 53 , and finally $\pi_{(q+3) n_{0}}\left(\bar{\kappa}_{G^{\prime}}\right) \subset \pi_{(q+2) n_{0}}\left(\widetilde{\mathrm{W}}_{q+2}^{\prime} \cap \widetilde{h}_{p, u}^{\prime}\left(F_{t+2}\right)\right)$.

Lemma 55. Fix $p, m, q, r, u, v \in \mathbb{N}$ with $\bar{h}\left(\mathcal{L}_{q n_{0}, r}^{\prime}\right) \prec \mathcal{L}_{p n_{0}, m} \prec \bar{h}\left(\mathcal{L}_{u n_{0}, v}^{\prime}\right)$. Let $t$ be the integer from Theorem 30. Let $k \in \mathbb{N}_{+}$and $G a(t+k)$-gap of $\mathcal{C}$. If $\widetilde{h}_{p, u}^{\prime}(G)$ is one $(q+k)$-gap then $\pi_{(t+k-1) n_{0}}(G) \subsetneq \mathbb{I}$ and $\pi_{(t+k) n_{0}}\left(\bar{\kappa}_{G}\right) \in$ $\pi_{(t+k-1) n_{0}}\left(\widetilde{\mathrm{W}}_{t+k-1} \cap \widetilde{h}_{q, p}\left(F_{q+k}^{\prime}\right)\right)$.

Proof. Note that if $\widetilde{h}_{q, p}\left(F_{q+1}^{\prime}\right)=F_{t}$, then by Corollary $39, \widetilde{h}_{p, u}(G)$ is a $(q+k+1)$-gap, and therefore the result is vacuously true. If $\widetilde{h}_{q, p}\left(F_{q+1}^{\prime}\right) \neq F_{t}$, the proof is similar to that of Lemma 54 .

Lemma 56 puts a limit on the "size" of the first gap when gaps do not go to gaps. 
Lemma 56. Fix $p, m, q, r, u, v \in \mathbb{N}$ with $\bar{h}\left(\mathcal{L}_{q n_{0}, r}^{\prime}\right) \prec \mathcal{L}_{p n_{0}, m} \prec \bar{h}\left(\mathcal{L}_{u n_{0}, v}^{\prime}\right)$. Let $t$ be the integer from Theorem 30. If $\widetilde{h}_{q, p}\left(F_{q+1}^{\prime}\right) \neq F_{t}$, then $\mathcal{I} \subset$ $\left(1-c_{n_{0}-1}, c_{n_{0}-1}\right)$ and $\mathcal{I}^{\prime} \subset\left(1-c_{n_{0}-1}^{\prime}, c_{n_{0}-1}^{\prime}\right)$.

Proof. Suppose $\widetilde{h}_{q, p}\left(F_{q+1}^{\prime}\right) \neq F_{t}$. Let $G^{\prime}$ be the first odd $(q+2)$-gap of $\mathcal{C}^{\prime}$. As $\widetilde{h}_{q, p}\left(F_{q+1}^{\prime}\right) \neq F_{t}, \widetilde{h}_{q, p}\left(G^{\prime}\right)$ is one $(t+1)$-gap [15, Lemma 81], and hence $\pi_{(q+1) n_{0}}\left(G^{\prime}\right) \subsetneq \mathbb{I}^{\prime}$ by Lemma 54 . Note $\pi_{(q+2) n_{0}}\left(G^{\prime}\right) \neq\left[c^{\prime}, c_{1}^{\prime}\right]$ as $g^{n_{0}}\left(\left[c^{\prime}, c_{1}^{\prime}\right]\right)=\mathbb{I}^{\prime}$. Let $L^{\prime}$ be the first leg of $G^{\prime}$ and $\left[c^{\prime}, c_{j}^{\prime}\right]=\pi_{(q+2) n_{0}}\left(L^{\prime}\right)$. Let $H^{\prime}$ be an arc of $\mathcal{C}^{\prime}$ such that $\pi_{(q+2) n_{0}}\left(H^{\prime}\right)=\pi_{(q+2) n_{0}+j}\left(L^{\prime}\right)$ and $\pi_{(q+2) n_{0}} \mid H^{\prime}$ is a homeomorphism. Set $\left\{\bar{x}^{\prime}, \bar{y}^{\prime}\right\}=\partial H^{\prime}$ with $\pi_{(q+2) n_{0}}\left(\bar{x}^{\prime}\right)=c^{\prime}$. Then $\bar{x}^{\prime}, \bar{y}^{\prime} \in \widetilde{\mathrm{W}}_{q+1}^{\prime}$ and $L_{q+1}\left(\bar{x}^{\prime}\right) \geq n_{0}$, and hence $\pi_{(q+1) n_{0}}\left(H^{\prime}\right)$ must contain the $(q+1) n_{0}$ projection of the first leg of $F_{q+1}$. Thus $\pi_{(q+1) n_{0}}\left(F_{q+1}^{\prime}\right) \subset$ $\pi_{(q+1) n_{0}}\left(H^{\prime}\right)$ (Lemma 48). Therefore, $\pi_{(q+1) n_{0}}\left(F_{(q+1) n_{0}}^{\prime}\right) \subset \pi_{(q+1) n_{0}+j}\left(G^{\prime}\right)$. If $\pi_{(q+1) n_{0}}\left(F_{(q+1) n_{0}}^{\prime}\right) \not \subset\left(1-c_{n_{0}-1}^{\prime}, c_{n_{0}-1}^{\prime}\right)$, then $\left[c^{\prime}, c_{1}^{\prime}\right] \subset \pi_{(q+1) n_{0}+j-1}\left(G^{\prime}\right)$, and hence $\mathbb{I}^{\prime}=\pi_{(q+1) n_{0}+j-2}\left(G^{\prime}\right)$, a contradiction as $j \geq 2$. Thus $\mathcal{I}^{\prime} \subset$ $\left(1-c_{n_{0}-1}^{\prime}, c_{n_{0}-1}^{\prime}\right)$. The proof for $\mathcal{I} \subset\left(1-c_{n_{0}-1}, c_{n_{0}-1}\right)$ is similar.

Lemma 57 forces the "size" of certain gaps.

Lemma 57. Fix $p \in \mathbb{N}$. Let $H$ be a p-gap of $\mathcal{C}$ and $G$ a p-gap of $\mathcal{C}$ whose center has p-level $n_{0}+1$. If $\pi_{p n_{0}}\left(\bar{\kappa}_{H}\right) \in \pi_{p n_{0}}\left(G \cap \widetilde{\mathrm{W}}_{p}\right)$, then $\pi_{p n_{0}}(H)=\left[c, c_{1}\right]$.

Proof. Suppose $\pi_{p n_{0}}\left(\bar{\kappa}_{H}\right) \in \pi_{p n_{0}}\left(G \cap \widetilde{\mathrm{W}}_{p}\right)$. Let $c_{j}=\pi_{p n_{0}}\left(\bar{\kappa}_{H}\right)$. Let $\bar{w}$ be the first $p$-wrapping point of $G$ such that $\pi_{p n_{0}}(\bar{w})=c_{j}$ and $J$ the arc that lies between the first $p$-wrapping point of $G$ and $\bar{w}$. Note that $J$ contains the first leg of $G$, and hence $\pi_{p n_{0}}(J)=\left[c, c_{1}\right]$ (Lemma 48). As $\pi_{p n_{0}+j}(\bar{w})=c$, we have $\pi_{p n_{0}+j}(J)=[c, x]$, where $x$ and $1-x$ are the numbers closest to $c$ for which $f^{j}(x)=c$. As $\pi_{p n_{0}+j}\left(\bar{\kappa}_{H}\right)=c$, either $[c, x]$ or $[c, 1-x]$ is contained in $\pi_{p n_{0}+j}(H)$. Thus, as $f^{j}$ is symmetric about $c, \pi_{p n_{0}}(H)=f^{j}\left(\pi_{p n_{0}+j}(H)\right) \supset$ $f^{j}\left(\pi_{p n_{0}+j}(J)\right)=\pi_{p n_{0}}(J)=\left[c, c_{1}\right]$. Hence $\pi_{p n_{0}}(H)=\left[c, c_{1}\right]$.

As outlined earlier, we now show gaps go to gaps.

TheOREM 58. Fix $p, m, q, r, u, v \in \mathbb{N}$ such that $\bar{h}\left(\mathcal{L}_{q n_{0}, r}^{\prime}\right) \prec \mathcal{L}_{p n_{0}, m} \prec$ $\bar{h}\left(\mathcal{L}_{u n_{0}, v}^{\prime}\right)$. Then there exists $t \in \mathbb{N}$ such that for every $k \in \mathbb{N}_{+}, \bar{h}_{q, p}\left(\widetilde{\Phi}_{q+k, 0}^{\prime}\right)$ $=\widetilde{\Phi}_{t+k-1,0}$. Alternatively stated, there exists $t \in \mathbb{N}$ such that for every $k \in \mathbb{N}$, any $(q+k)$-gap of $\mathcal{C}^{\prime}$ maps under $\widetilde{h}_{q, p}$ to exactly one $(t+k-1)$-gap of $\mathcal{C}$.

Proof. If $\widetilde{h}_{q, p}\left(F_{q+1}^{\prime}\right)=F_{t}$, then Corollary 39 gives us $\bar{h}_{q, p}\left(\widetilde{\Phi}_{q+k, 0}^{\prime}\right)=$ $\widetilde{\Phi}_{t+k-1,0}$ for all $k \in \mathbb{N}_{+}$. We prove $\widetilde{h}_{q, p}\left(F_{q+1}^{\prime}\right)=F_{t}$ by contradiction.

Let $t$ be the integer from Theorem 30 and assume $\widetilde{h}_{q, p}\left(F_{q+1}^{\prime}\right) \neq F_{t}$. Let $G^{\prime}$ be a $(q+2)$-gap of $\mathcal{C}^{\prime}$ with $L_{q+2}\left(\bar{\kappa}_{G^{\prime}}\right)=n_{0}+1$. We begin by showing that

$$
\left|\pi_{(q+2) n_{0}}\left(\widetilde{h}_{p, u}^{\prime}\left(F_{t+2}\right) \cap \widetilde{\mathrm{W}}_{q+2}^{\prime}\right)\right|<\left|\pi_{(q+2) n_{0}}\left(G^{\prime} \cap \widetilde{\mathrm{W}}_{q+2}^{\prime}\right)\right|,
$$


or in other words, $\widetilde{h}_{p, u}^{\prime}\left(F_{t+2}\right)$ turns at fewer end points than $G^{\prime}$ (see Example 1.12). By Lemma $53, \pi_{(q+2) n_{0}}\left(\widetilde{h}_{p, u}^{\prime}\left(F_{t+2}\right)\right) \subset \mathcal{I}^{\prime}$, and by Lemma 56 , $\mathcal{I}^{\prime} \subset\left(1-c_{n_{0}-1}^{\prime}, c_{n_{0}-1}^{\prime}\right)$. Hence $\pi_{(q+2) n_{0}}\left(\widetilde{h}_{p, u}^{\prime}\left(F_{t+2}\right)\right) \subset\left(1-c_{n_{0}-1}^{\prime}, c_{n_{0}-1}^{\prime}\right)$. Thus $g\left(\pi_{(q+2) n_{0}}\left(\widetilde{h}_{p, u}^{\prime}\left(F_{t+2}\right)\right)\right) \subset\left(c^{\prime}, c_{1}^{\prime}\right]$. By the choice of $G^{\prime}, \pi_{(q+2) n_{0}}\left(G^{\prime}\right)=$ $\left[c^{\prime}, c_{1}^{\prime}\right], \pi_{(q+3) n_{0}+1}\left(\bar{\kappa}_{G}^{\prime}\right)=c^{\prime}$, and $\pi_{(q+3) n_{0}+1} \mid G^{\prime}$ is a homeomorphism. Thus, as $g\left(\pi_{(q+2) n_{0}}\left(\widetilde{h}_{p, u}^{\prime}\left(F_{t+2}\right)\right)\right) \subset\left(c^{\prime}, c_{1}^{\prime}\right] \subsetneq\left[c^{\prime}, c_{1}^{\prime}\right]=\pi_{(q+2) n_{0}}\left(G^{\prime}\right)$ and $c^{\prime}$ is in both $\pi_{(q+3) n_{0}}\left(\widetilde{h}_{p, u}^{\prime}\left(F_{t+2}\right)\right)$ and $\pi_{(q+3) n_{0}+1}\left(G^{\prime}\right)$, it follows that $\pi_{(q+3) n_{0}}\left(\widetilde{h}_{p, u}^{\prime}\left(F_{t+2}\right)\right)$ $\subsetneq \pi_{(q+3) n_{0}+1}\left(G^{\prime}\right)$. Hence

$$
g\left(\pi_{(q+2) n_{0}}\left(\widetilde{h}_{p, u}^{\prime}\left(F_{t+2}\right) \cap \widetilde{\mathrm{W}}_{q+2}^{\prime}\right)\right) \subsetneq \pi_{(q+2) n_{0}}\left(G^{\prime} \cap \widetilde{\mathrm{W}}_{q+2}^{\prime}\right) .
$$

Therefore, $\left|\pi_{(q+2) n_{0}}\left(\widetilde{h}_{p, u}^{\prime}\left(F_{t+2}\right) \cap \widetilde{\mathrm{W}}_{q+2}^{\prime}\right)\right|<\left|\pi_{(q+2) n_{0}}\left(G^{\prime} \cap \widetilde{\mathrm{W}}_{q+2}^{\prime}\right)\right|$.

We next show that

$$
\left|\pi_{(q+2) n_{0}}\left(G^{\prime} \cap \widetilde{\mathrm{W}}_{q+2}^{\prime}\right)\right| \leq\left|\pi_{(t+1) n_{0}}\left(\widetilde{h}_{q, p}\left(F_{q+2}^{\prime}\right) \cap \widetilde{\mathrm{W}}_{t+1}\right)\right|,
$$

or in other words, $G^{\prime}$ turns at fewer end points than $\widetilde{h}_{q, p}\left(F_{q+2}^{\prime}\right)$. Let $c_{j}^{\prime} \in$ $\pi_{(q+2) n_{0}}\left(G^{\prime} \cap{\widetilde{\mathrm{W}^{\prime}}}_{q+2}^{\prime}\right), 0<j<n_{0}$. By Lemma 46 , there exists a $(q+3)$-gap, say $H^{\prime}$, for which $\pi_{(q+3) n_{0}}\left(\bar{\kappa}_{H^{\prime}}\right)=c_{j}$. By Lemma $57, \pi_{(q+3) n_{0}}\left(H^{\prime}\right)=\left[c, c_{1}\right]$, and hence $\pi_{(q+3) n_{0}}\left(H^{\prime}\right) \not \subset \mathcal{I}^{\prime}$ by Lemma 56 . Thus $\overrightarrow{\mathcal{S}}_{q+3}\left(H^{\prime}\right)$ is more than one $(t+2)$-gap by Lemma 49 . Let $K$ be the $(t+2)$-gap such that $\bar{h}_{q, p}\left(\bar{\kappa}_{H^{\prime}}\right) \in K$ and $J$ the first $(t+2)$-gap such that $K \triangleleft J$. Note that $K, J \in \overrightarrow{\mathcal{S}}_{q+3}\left(H^{\prime}\right)$. Let $Y^{\prime}$ be the $(q+2)$-gap such that $\bar{\kappa}_{H^{\prime}} \in Y^{\prime}$. Thus $Y^{\prime} \subset \widetilde{h}_{p, u}^{\prime}(K)$ and $\bar{h}_{p, u}^{\prime}\left(\bar{\kappa}_{K}\right)$ $=\bar{\kappa}_{Y^{\prime}}$. Additionally, as $\pi_{(q+2) n_{0}}\left(Y^{\prime}\right)=\left[c, c_{1}^{\prime}\right]$ (Lemma 57), $\overrightarrow{\mathcal{S}}_{q+2}\left(Y^{\prime}\right)$ contains more than one $(t+1)$-gap (Lemmas 56 and 49). Suppose $Y^{\prime} \neq \widetilde{h}_{p, u}^{\prime}(K)$. By [15, Lemma 81], each odd $(q+2)$-gap of $\overrightarrow{\mathcal{S}}_{t+2}^{\prime}(K)$ maps to one $(t+1)$-gap under $\widetilde{h}_{q, p}$. Hence $Y^{\prime}$ is even, and therefore $K$ is even by Lemma 45 . It follows that $J$ is odd and $\widetilde{h}_{p, u}^{\prime}(J)$ is one $(q+2)$-gap [15, Lemma 81]. Thus $\overrightarrow{\mathcal{S}}_{q+2}\left(\widetilde{h}_{p, u}^{\prime}(J)\right)$ is more than one $(t+1)$-gap. Therefore, by Lemma 49 , if $M^{\prime} \in{\overrightarrow{\mathcal{S}_{t+2}^{\prime}}}^{\prime}(K)$ is an odd gap, then $\pi_{(q+2) n_{0}}\left(M^{\prime}\right) \subsetneq \pi_{(q+2) n_{0}}\left(\widetilde{h}_{p, u}^{\prime}(J)\right)$ and $\pi_{(q+2) n_{0}}\left(M^{\prime}\right) \subsetneq \mathcal{I}^{\prime}$. Let $L^{\prime}$ be the first leg of $\widetilde{h}_{p, u}^{\prime}(J)$ and $\left\{\bar{w}_{0}^{\prime}, \bar{w}_{1}^{\prime}, \ldots, \bar{w}_{d}^{\prime}\right\}=$ $\mathrm{W}_{q+2}^{\prime} \cap\left\{L^{\prime} \cup \widetilde{h}_{p, u}^{\prime}(K)\right\}$. Let $i=\sup \left\{k \mid k<d\right.$ and $\left.\pi_{(q+2) n_{0}}\left(\bar{w}_{k}^{\prime}\right) \notin \mathcal{I}^{\prime}\right\}$. As $\pi_{(q+2) n_{0}}\left(Y^{\prime}\right)=\left[c, c_{1}^{\prime}\right], i$ exists. Furthermore, $\bar{w}_{i}^{\prime}$ is an even $(q+2)$-wrapping point and $\bar{w}_{i}^{\prime}$ is not in the last $(q+2)$-gap of $\overrightarrow{\mathcal{S}}_{t+2}(K)$ as that gap is type $\varphi^{\prime}$ [15, Proposition 80]. Let $\bar{w}_{e}^{\prime}$ be the $(q+2)$-wrapping point such that $L_{q+2}\left(\bar{w}_{e}^{\prime}\right) \geq L_{q+2}\left(\bar{w}_{k}^{\prime}\right)$ for all $i<k<d$. Note that $L_{q+2}\left(\bar{w}_{e}\right) \geq \varphi^{\prime}$. By Proposition 23, either $\bar{w}_{i}^{\prime}$ or $\bar{w}_{d}^{\prime}$ is contained in a $(q+2)$-symmetry about $\bar{w}_{e}^{\prime}$. However, by the choice of $\bar{w}_{i}^{\prime}$ and $\bar{w}_{d}^{\prime}$ neither can be in a $(q+2)$ symmetry about $\bar{w}_{e}^{\prime}$, a contradiction. Thus $Y^{\prime}=\widetilde{h}_{p, u}^{\prime}(K)$. Therefore, by Lemma $55, \pi_{(t+2) n_{0}}\left(\bar{\kappa}_{K}\right) \in \pi_{(t+1) n_{0}}\left(\widetilde{h}_{q, p}\left(F_{q+2}^{\prime}\right) \cap \widetilde{\mathrm{W}}_{t+1}\right)$. Hence, for every $c_{j}^{\prime} \in$ 
$\pi_{(q+2) n_{0}}\left(G^{\prime} \cap \widetilde{\mathrm{W}}_{q+2}^{\prime}\right)$, we have $\pi_{(t+1) n_{0}}\left(\bar{h}\left(\bar{c}_{j}^{\prime}\right)\right) \in \pi_{(t+1) n_{0}}\left(\widetilde{h}_{q, p}\left(F_{q+2}^{\prime} \cap \widetilde{\mathrm{W}}_{t+1}\right)\right)$. Thus $\left|\pi_{(q+2) n_{0}}\left(G^{\prime} \cap \widetilde{\mathrm{W}}_{q+2}^{\prime}\right)\right| \leq\left|\pi_{(t+1) n_{0}}\left(\widetilde{h}_{q, p}\left(F_{q+2}^{\prime}\right) \cap \widetilde{\mathrm{W}}_{t+1}\right)\right|$.

Let $G$ be a $(t+1)$-gap of $\mathcal{C}$ with a center of $(t+1)$-level $n_{0}+1$. By an argument similar to the one in the second paragraph of this proof,

$$
\left|\pi_{(t+1) n_{0}}\left(\widetilde{h}_{q, p}\left(F_{q+2}^{\prime}\right) \cap \widetilde{\mathrm{W}}_{t+1}\right)\right|<\left|\pi_{(t+1) n_{0}}\left(G \cap \widetilde{\mathrm{W}}_{t+1}\right)\right| .
$$

In other words, $\widetilde{h}_{q, p}\left(F_{q+2}^{\prime}\right)$ turns at fewer end points than $G$. By an argument similar to the third paragraph of this proof,

$$
\left|\pi_{(t+1) n_{0}}\left(G \cap \widetilde{\mathrm{W}}_{t+1}\right)\right| \leq\left|\pi_{(q+1) n_{0}}\left(\widetilde{h}_{p, u}^{\prime}\left(F_{t+1}\right) \cap \widetilde{\mathrm{W}}_{q+1}^{\prime}\right)\right| .
$$

In other words, $G$ turns at fewer end points than $\widetilde{h}_{p, u}^{\prime}\left(F_{t+1}\right)$. Hence $\widetilde{h}_{p, u}^{\prime}\left(F_{t+2}\right)$ turns at fewer end points than $G^{\prime}$ which turns at fewer end points than $\widetilde{h}_{q, p}\left(F_{q+2}^{\prime}\right)$ which turns at fewer end points than $G$ which turns at fewer end points than $\widetilde{h}_{p, u}^{\prime}\left(F_{t+1}\right)$. Thus $\widetilde{h}_{p, u}^{\prime}\left(F_{t+2}\right)$ turns at fewer end points than $\widetilde{h}_{p, u}^{\prime}\left(F_{t+1}\right)$, or

$$
\left|\pi_{(q+2) n_{0}}\left(\widetilde{h}_{p, u}^{\prime}\left(F_{t+2}\right) \cap \widetilde{\mathrm{W}}_{q+2}^{\prime}\right)\right|<\left|\pi_{(q+1) n_{0}}\left(\widetilde{h}_{p, u}^{\prime}\left(F_{t+1}\right) \cap \widetilde{\mathrm{W}}_{q+1}^{\prime}\right)\right| .
$$

However, by Proposition $42, \overrightarrow{\mathcal{S}}_{t+2}\left(F_{t+2}\right) \sim \overrightarrow{\mathcal{S}}_{t+1}\left(F_{t+1}\right)$, and hence $\widetilde{h}_{q, p}\left(F_{q+2}^{\prime}\right)$ and $\widetilde{h}_{p, u}^{\prime}\left(F_{t+1}\right)$ turn at the same number of end points, or

$$
\left|\pi_{(q+2) n_{0}}\left(\widetilde{h}_{p, u}^{\prime}\left(F_{t+2}\right) \cap \widetilde{\mathrm{W}}_{q+2}^{\prime}\right)\right|=\left|\pi_{(q+1) n_{0}}\left(\widetilde{h}_{p, u}^{\prime}\left(F_{t+1}\right) \cap \widetilde{\mathrm{W}}_{q+1}^{\prime}\right)\right|,
$$

a contradiction.

In Lemma 59 we show that the image of a type $2(q+k)$-gap of $\mathcal{C}^{\prime}$ under $\widetilde{h}_{q, p}$ will have only 2 legs. Similarly, in Lemma 60 we show that the image of a type $2(t+k)$-gap of $\mathcal{C}$ under $\widetilde{h}_{p, u}^{\prime}$ has only 2 legs.

LEMmA 59. Fix $p, m, q, r, u, v \in \mathbb{N}$ with $\bar{h}\left(\mathcal{L}_{q n_{0}, r}^{\prime}\right) \prec \mathcal{L}_{p n_{0}, m} \prec \bar{h}\left(\mathcal{L}_{u n_{0}, v}^{\prime}\right)$. Let $t$ be the integer from Theorem 58. Let $k \in \mathbb{N}_{+}$and $G^{\prime} a(q+k)$-gap of $\mathcal{C}^{\prime}$ such that $T\left(G^{\prime}\right)=2$. Then $\partial\left(\widetilde{h}_{q, p}\left(G^{\prime}\right)\right)$ and $\bar{\kappa}_{\widetilde{h}_{q, p}\left(G^{\prime}\right)}$ are the only $(t+k-1)$ wrapping points contained in $\widetilde{h}_{q, p}\left(G^{\prime}\right)$.

Proof. Define $H=\widetilde{h}_{q, p}\left(G^{\prime}\right)$ and let $L^{\prime}$ be the first leg of $G^{\prime}$. As $T\left(G^{\prime}\right)=2$, $\partial G^{\prime}$ and $\bar{\kappa}_{G^{\prime}}$ are the only $(q+k)$-wrapping points in $G^{\prime}$. Hence $L^{\prime}$ is the arc that lies between the first $(q+k)$-wrapping point of $G^{\prime}$ and $\bar{\kappa}_{G^{\prime}}$, and $\pi_{(q+k) n_{0}}\left(L^{\prime}\right)=\left[c_{2}^{\prime}, c^{\prime}\right]$. Furthermore, $\widetilde{h}_{q, p}\left(L^{\prime}\right)$ is the arc that lies between the first $(t+k-1)$-wrapping point of $H$ and $\bar{\kappa}_{H}$. Thus $\widetilde{h}_{q, p}\left(L^{\prime}\right)$ contains all $p$-forced symmetries for any $p$-wrapping point contained in the interior of $\widetilde{h}_{q, p}\left(L^{\prime}\right)$.

Let $J^{\prime}$ be a $(q+k)$-gap of $\mathcal{C}^{\prime}$ such that $T\left(J^{\prime}\right)=n_{0}+1$ and let $K^{\prime}$ be the first leg of $J^{\prime}$. Note that $\pi_{(q+k) n_{0}}\left(K^{\prime}\right)=\left[c^{\prime}, c_{1}^{\prime}\right]$ by Lemma 48. By Proposition 29, the symmetry of the map $g$, and Theorem 27 , if $\bar{w} \in \widetilde{h}_{q, p}\left(\stackrel{\circ}{L}^{\prime}\right) \cap \widetilde{\mathrm{W}}_{t+k-1}$ then 
there exists $\bar{v} \in \widetilde{h}_{q, p}\left(K^{\prime}\right) \cap \widetilde{\mathrm{W}}_{t+k-1}$ such that $\pi_{t+k-1}(\bar{w})=\pi_{t+k-1}(\bar{v})$. Furthermore, as $G^{\prime}$ and $J^{\prime}$ have opposite orientations, $\widetilde{h}_{q, p}\left(G^{\prime}\right)$ and $\widetilde{h}_{q, p}\left(J^{\prime}\right)$ will also have opposite orientations (Lemma 45). Thus any $(t+k-1)$-wrapping point contained in the interior of $\widetilde{h}_{q, p}\left(G^{\prime}\right)$ would have opposite orientation to any $(t+k-1)$-wrapping point contained in the interior of $\widetilde{h}_{q, p}\left(J^{\prime}\right)$. Hence the interior of $\widetilde{h}_{q, p}\left(L^{\prime}\right)$ contains no $(t+k-1)$-wrapping points.

Lemma 60. Fix $p, m, q, r, u, v \in \mathbb{N}$ with $\bar{h}\left(\mathcal{L}_{q n_{0}, r}^{\prime}\right) \prec \mathcal{L}_{p n_{0}, m} \prec \bar{h}\left(\mathcal{L}_{u n_{0}, v}^{\prime}\right)$. Let $t$ be the integer from Theorem 58. Let $k \in \mathbb{N}$ and $G a(t+k)$-gap of $\mathcal{C}$ such that $T(G)=2$. Then $\partial\left(\widetilde{h}_{p, u}^{\prime}(G)\right)$ and $\bar{\kappa}_{\widetilde{h}_{p, u}^{\prime}(G)}$ are the only $(q+k+1)$ wrapping points contained in $\widetilde{h}_{p, u}^{\prime}(G)$.

Proof. Similar to that of Lemma 59.

In Lemma 61 we show that if $f$ and $g$ have different kneading sequences, then the number of $(q+1)$-gaps in a type $2(q+2)$-gap of $\mathcal{C}^{\prime}$ is different than the number of $t$-gaps in a type $2(t+1)$-gap of $\mathcal{C}$.

Lemma 61. Let $q, t \in \mathbb{N}_{+}$. Let $G^{\prime}$ be a $(q+2)$-gap of $\mathcal{C}^{\prime}$ such that $T\left(G^{\prime}\right)$ $=2$, and $H a(t+1)$-gap of $\mathcal{C}$ such that $T(H)=2$. If $K(f)<K(g)$ in the parity-lexicographical ordering, then the number of t-gaps in $H$ is less than the number of $(q+1)$-gaps in $G^{\prime}$.

Proof. The intuition behind this proof is that the lap number increases as the kneading sequence increases. Let $P=\left\{x \in\left(c_{2}, c\right) \mid f^{n_{0}}(x)=c\right\}$. As $H$ is a type 2 gap, it has exactly 2 legs and the $p n_{0}$ th projection of each leg is $\left[c_{2}, c\right]$. Hence, the number of $t$-gaps in $H$ is exactly $2|P|+1$. Let $x \in\left(c_{2}, c\right)$. Then $x \in P$ if and only if the following conditions hold:

(i) $I_{f}(x)=L b_{1} \ldots b_{n_{0}-1} C$ where $b_{i} \in\{L, R\}$ for all $0<i<n_{0}$,

(ii) $I_{f}(x)>I_{f}\left(c_{2}\right)$ in the parity-lexicographical ordering, and

(iii) $b_{i} b_{i+1} \ldots b_{n_{0}-1} C<K(f)$ in this ordering for all $0<i<n_{0}$.

Furthermore, if $J$ is a sequence of $R$ 's, $L$ 's and $C$ 's that satisfy these conditions, then there exists $x \in P$ such that $I_{f}(x)=J$. Similar statements can be made about the $(q+2)$-gap $G^{\prime}$ and the set $Q=\left\{x \in\left(c_{2}^{\prime}, c^{\prime}\right) \mid\right.$ $\left.g^{n_{0}}(x)=c^{\prime}\right\}$.

Suppose $K(f)<K(g)$ in the parity-lexicographical ordering. Then $I_{f}\left(c_{1}\right)$ $<I_{g}\left(c_{1}^{\prime}\right)$ and $I_{f}\left(c_{2}\right)>I_{g}\left(c_{2}^{\prime}\right)$ in this ordering. Hence for each $x \in P$ there exists $z \in Q$ such that $I_{g}(z)=I_{f}(x)$. Thus $|P| \leq|Q|$. Consider the sequence $L K(f)$. As $K(f) \nless K(f)$, there does not exist an $x \in P$ such that $I_{f}(x)=$ $L K(f)$. However, there does exist $x \in Q$ such that $I_{g}(x)=L K(f)$, thus $|P|<|Q|$.

In Lemma 62 we show the existence of specific types of gaps in certain kinds of arcs. 
Lemma 62. Fix $p \in \mathbb{N}$. Let $\bar{x}, \bar{y} \in \widetilde{\mathrm{W}}_{p}$ be such that $L_{p}(\bar{x})=k n_{0}+j$ and $L_{p}(\bar{y})=i n_{0}+j+1$ where $k, i \in \mathbb{N}$ and $0 \leq j<n_{0}$. Then for every $n<\min \{k, i\} n_{0}+j$ there exists $\bar{z} \in \widetilde{\mathrm{W}}_{p}$ between $\bar{x}$ and $\bar{y}$ such that $L_{p}(\bar{z})=n$. Furthermore, either there exists a p-gap $G$ that lies between $\bar{x}$ and $\bar{y}$ such that $L_{p}\left(\bar{\kappa}_{G_{n}}\right)=n$ or there is no p-gap whose center has p-level $n$.

Proof. It is sufficient to show the conclusion for $n=\min \{k, i\} n_{0}+j-1$. Let $m=\min \{k, i\}$. Without loss of generality assume $\bar{x} \triangleleft \bar{y}$. Let $\bar{x}^{\prime}$ be the closest $p$-wrapping point to $\bar{y}$ such that $\bar{x}^{\prime} \triangleleft \bar{y}, L_{p}\left(\bar{x}^{\prime}\right) \geq m n_{0}+j$, and $L_{p}\left(\bar{x}^{\prime}\right) \bmod n_{0}=j$. Let $\bar{y}^{\prime}$ be the closest $p$-wrapping point to $\bar{x}^{\prime}$ such that $\bar{x}^{\prime} \triangleleft \bar{y}^{\prime}, L_{p}\left(\bar{y}^{\prime}\right) \geq m n_{0}+j+1$, and $L_{p}\left(\bar{y}^{\prime}\right) \bmod n_{0}=j+1$. Let $H$ be the arc that lies between $\bar{x}^{\prime}$ and $\bar{y}^{\prime}$. By Propositions 23 and 24, if $\bar{z}$ is a $p$-wrapping point in the interior of $H$, then $L_{p}(\bar{z})<m n_{0}+j$. Thus $\pi_{m n_{0}+j-1} \mid H$ is a homeomorphism and $\pi_{m n_{0}+j-1}(H)=\mathbb{I}$. Hence there exists $\bar{z} \in H$ such that $\pi_{m n_{0}+j-1}(\bar{z})=c$, and therefore $L_{p}(\bar{z})=m n_{0}+j-1$. The last statement follows from Lemma 46.

We can now prove our main result.

THEOREM 63. Let $f, g$ be tent maps with periodic critical points. If $K(f)$ $\neq K(g)$, then $(\mathbb{I}, f)$ and $\left(\mathbb{I}^{\prime}, g\right)$ are not homeomorphic.

Proof. Let $K(f) \neq K(g)$ and $n_{0}$ be the period of the critical point for both $f$ and $g$. (Note that if the period of the critical points is different for the two maps, then $(\mathbb{I}, f)$ and $\left(\mathbb{I}^{\prime}, g\right)$ will have a different number of end points.) Assume $(\mathbb{I}, f)$ and $\left(\mathbb{I}^{\prime}, g\right)$ are homeomorphic and $h:\left(\mathbb{I}^{\prime}, g\right) \rightarrow(\mathbb{I}, f)$ is a homeomorphism. We can find $p, m, q, r, u, v \in \mathbb{N}$ such that $\bar{h}\left(\mathcal{L}_{q n_{0}, r}^{\prime}\right) \prec$ $\mathcal{L}_{p n_{0}, m} \prec \bar{h}\left(\mathcal{L}_{u n_{0}, v}^{\prime}\right)$ (Lemma 11(ii)). Let $t$ be the integer from Theorem 58 . If $H$ is a $(t+k+1)$-gap of $\mathcal{C}$, let

$$
\eta(H)=\left\{c_{i} \mid \exists J \in H \text { such that } J \text { is a }(t+k) \text {-gap and } \pi_{(t+k) n_{0}}\left(\bar{\kappa}_{J}\right)=c_{i}\right\} .
$$

We now consider the three possible cases.

CAse 1: $K(f)=R L L \ldots C$ and $K(g)=R L L \ldots C$. By Lemma 46, for every $i \in \mathbb{N}$ such that $i \bmod n_{0} \neq 0$ there exists a $(q+2)$-gap $G^{\prime}$ of $\mathcal{C}^{\prime}$ and a $(t+1)$-gap $H$ of $\mathcal{C}$ such that $L_{q+2}\left(\bar{\kappa}_{G^{\prime}}\right)=i=L_{p+1}\left(\bar{\kappa}_{H}\right)$. Let $G^{\prime}$ be a $(q+2)$ gap of $\mathcal{C}^{\prime}$ such that $T\left(G^{\prime}\right)=1$, and compare the $(q+1)$-gaps contained in $G^{\prime}$ and the $t$-gaps contained in $\widetilde{h}_{q, p}\left(G^{\prime}\right)$. By Lemma $62, \eta^{\prime}\left(G^{\prime}\right)=\left\{c_{i}^{\prime} \mid 0<\right.$ $\left.i<n_{0}\right\}$. Thus by Theorem 58, $\eta\left(\widetilde{h}_{q, p}\left(G^{\prime}\right)\right)=\left\{c_{i} \mid 0<i<n_{0}\right\}$. Let $J^{\prime}$ be the first $(q+1)$-gap in $G^{\prime}$ such that $\pi_{(q+1) n_{0}}\left(\bar{\kappa}_{J^{\prime}}\right)=c_{n_{0}-1}^{\prime}$. By Lemma 62, for all $0<i<n_{0}-1$ there exists a $(q+1)$-gap $K^{\prime} \subset G^{\prime}$ such that $K^{\prime} \triangleleft J^{\prime}$ and $\pi_{(q+1) n_{0}}\left(\bar{\kappa}_{K^{\prime}}\right)=c_{i}^{\prime}$. Similarly, if $H$ is the first $t$-gap of $\widetilde{h}_{q, p}\left(G^{\prime}\right)$ such that $\pi_{t n_{0}}(H)=c_{n_{0}-1}$, then for all $0<i<n_{0}-1$ there exists a $t$-gap $K \subset \widetilde{h}_{q, p}\left(G^{\prime}\right)$ such that $K \triangleleft H$ and $\pi_{t n_{0}}(H)=c_{i}$. Hence $\bar{h}\left(\bar{c}_{n_{0}-1}^{\prime}\right)=\bar{c}_{n_{0}-1}$. 
Let $M^{\prime}$ be the last $(q+1)$-gap such that $M^{\prime} \triangleleft J^{\prime}$ and $\pi_{(q+1) n_{0}}\left(M^{\prime}\right)=c_{n_{0}-2}^{\prime}$. By Lemma 62 , for all $0<i<n_{0}-2$ there exists a $(q+1)$-gap $K^{\prime} \subset G^{\prime}$ such that $M^{\prime} \triangleleft K^{\prime} \triangleleft J^{\prime}$ and $\pi_{(q+1) n_{0}}\left(\bar{\kappa}_{K^{\prime}}\right)=c_{i}^{\prime}$. Similarly, if $H$ is the last $t$-gap such that $H \triangleleft \widetilde{h}_{q, p}\left(J^{\prime}\right)$ and $\pi_{t n_{0}}(H)=c_{n_{0}-2}$, then for all $0<i<n_{0}-2$ there exists a $t$-gap $K \subset \widetilde{h}_{q, p}\left(G^{\prime}\right)$ such that $H \triangleleft K \triangleleft \widetilde{h}_{q, p}\left(J^{\prime}\right)$ and $\pi_{t n_{0}}(H)=c_{i}$. Hence $\bar{h}\left(\bar{c}_{n_{0}-2}^{\prime}\right)=\bar{c}_{n_{0}-2}$. Continuing in this manner, we have $\bar{h}\left(\bar{c}_{i}^{\prime}\right)=\bar{c}_{i}$ for all $0 \leq i<n_{0}$. Hence, by Lemma $45, K(f)=K(g)$, a contradiction. Thus $(\mathbb{I}, f)$ and $\left(\mathbb{I}^{\prime}, g\right)$ are not homeomorphic.

CASE 2: $K(f)=R L R R \ldots C$ and $K(g)=R L L \ldots C$. Let $G^{\prime}$ be a $(q+2)$ gap of $\mathcal{C}^{\prime}$ such that $T\left(G^{\prime}\right)=2$. Suppose there exists a $(q+1)$-gap $J^{\prime} \subset G^{\prime}$ such that $\pi_{(q+1) n_{0}}\left(\bar{\kappa}_{J^{\prime}}\right)=c_{n_{0}-1}^{\prime}$. By Lemma $62, \eta^{\prime}\left(G^{\prime}\right)=\left\{c_{i}^{\prime} \mid 0<i<n_{0}\right\}$. Thus $\eta\left(\widetilde{h}_{q, p}\left(G^{\prime}\right)\right)=\left\{c_{i} \mid 0<i<n_{0}\right\}$. However, by Lemma 46, there is no $t$-gap of $\mathcal{C}$ whose center has a $t$-level of 1 . Thus, the center of a $t$-gap $J \subset \widetilde{h}_{q, p}\left(G^{\prime}\right)$ such that $\pi_{t n_{0}}\left(\bar{\kappa}_{J}\right)=c_{1}$ must have a $t$-level greater than or equal to $n_{0}+1$. Thus $\bar{\kappa}_{J} \in \widetilde{\mathrm{W}}_{t+1}$, a contradiction to Lemma 59 . Hence there does not exist a $(q+1)$-gap $J^{\prime} \subset G^{\prime}$ such that $\pi_{(q+1) n_{0}}\left(\bar{\kappa}_{J^{\prime}}\right)=c_{n_{0}-1}^{\prime}$.

As $T\left(G^{\prime}\right)=2, \pi_{(q+2) n_{0}}\left(G^{\prime}\right)=\left[c_{2}^{\prime}, c^{\prime}\right]$. Hence $\pi_{(q+2) n_{0}-1}\left(G^{\prime}\right)=\left[c_{3}^{\prime}, c_{1}^{\prime}\right]$. As $c_{3}^{\prime} \in \mathbb{I}_{L}$, there exists $\bar{y}^{\prime} \in G^{\prime}$ such that $\pi_{(q+2) n_{0}-1}\left(\bar{y}^{\prime}\right)=c^{\prime}$, and therefore $\bar{y}^{\prime} \in \widetilde{\mathrm{W}}_{q+1}$ and $L_{q+1}\left(\bar{y}^{\prime}\right)=n_{0}-1$. Note that as $(q+1)$-gaps are $(q+1)$ symmetric, $\bar{y}$ is in the same $(q+1)$-gap as $\bar{\kappa}_{G^{\prime}}$, and hence $\bar{c}_{n_{0}}^{\prime}$ and $\bar{c}_{2}^{\prime}$ have the same orientation. By Lemma $62, \eta^{\prime}\left(G^{\prime}\right)=\left\{c_{i}^{\prime} \mid 0<i<n_{0}-1\right\}$. By Lemmas 59 and 46, $c_{1} \notin\left\{c_{i} \mid \exists J \subset \widetilde{h}_{q, p}\left(G^{\prime}\right)\right.$ such that $J$ is a $t$-gap and $\left.\pi_{t n_{0}}\left(\bar{\kappa}_{J}\right)=c_{i}\right\}$. Therefore $\eta\left(\widetilde{h}_{q, p}\left(G^{\prime}\right)\right)=\left\{c_{i} \mid 1<i<n_{0}\right\}$. Hence $\bar{h}\left(\bar{c}_{n_{0}-1}^{\prime}\right)=\bar{c}_{1}$, and therefore, by Corollary $45, \bar{h}\left(\bar{c}_{2}^{\prime}\right)$ and $\bar{c}_{1}$ have the same orientation.

Let $J^{\prime}$ be a $(q+2)$-gap of $\mathcal{C}^{\prime}$ such that $T\left(J^{\prime}\right)=1$. By Lemma 62, there exists a $(q+1)$-gap $K^{\prime} \subset J^{\prime}$ such that $L_{q+1}\left(\bar{\kappa}_{K^{\prime}}\right)=n_{0}-1$. As $\bar{h}\left(\bar{c}_{n_{0}-1}^{\prime}\right)=\bar{c}_{1}$ and there is not a type 1 gap of $\mathcal{C}, L_{t}\left(\bar{h}_{q, p}\left(\bar{\kappa}_{K^{\prime}}\right)\right)=1+k n_{0}$ for some $k \in \mathbb{N}_{+}$. This implies $\bar{c}_{1}$ and $\bar{h}\left(\bar{c}_{1}^{\prime}\right)$ have the same orientation. Hence $\bar{c}_{1}$ has the same orientation as both $\bar{h}\left(\bar{c}_{2}^{\prime}\right)$ and $\bar{h}\left(\bar{c}_{1}^{\prime}\right)$, which have opposite orientations, a contradiction. Thus $(\mathbb{I}, f)$ and $\left(\mathbb{I}^{\prime}, g\right)$ are not homeomorphic.

CASe 3: $K(f)=R L R R \ldots C$ and $K(g)=R L R R \ldots C$. Assume without loss of generality that $K(f)<K(g)$ in the parity-lexicographical ordering. As kneading sequences are shift maximal, there are more $R$ 's than $L$ 's in both $K(f)$ and $K(g)$. Thus, by Corollary 45, up end points map only to up end points. In particular, $\pi_{t n_{0}}\left(\bar{h}\left(\bar{c}_{2}^{\prime}\right)\right)<c$. Hence, by Lemmas 59 and $60, \bar{h}\left(\bar{c}_{2}^{\prime}\right)=\bar{c}_{2}$. Let $G^{\prime}$ be a $(q+2)$-gap of $\mathcal{C}^{\prime}$ with $T\left(G^{\prime}\right)=2$. Then $T\left(\widetilde{h}_{q, p}\left(G^{\prime}\right)\right)=2$. However, the number of $t$-gaps in $\widetilde{h}_{q, p}\left(G^{\prime}\right)$ is less than the number of $(q+1)$-gaps in $G^{\prime}$ (Lemma 61$)$, a contradiction to Theorem 58 . Thus $(\mathbb{I}, f)$ and $\left(\mathbb{I}^{\prime}, g\right)$ are not homeomorphic. 


\section{References}

[1] J. M. Aarts and R. J. Fokkink, The classification of solenoids, Proc. Amer. Math. Soc. 111 (1991), 1161-1163.

[2] M. Barge, Horseshoe maps and inverse limits, Pacific J. Math. 121 (1986), 29-39.

[3] M. Barge, K. Brucks and B. Diamond, Self-similarity in inverse limit spaces of the tent family, Proc. Amer. Math. Soc. 124 (1996), 3563-3570.

[4] M. Barge and B. Diamond, Homeomorphisms of inverse limit spaces of one-dimensional maps, Fund. Math. 146 (1995), 171-187.

[5] - - - Inverse limit spaces of infinitely renormalizable maps, Topology Appl. 83 (1998), 103-108.

[6] M. Barge and S. Holte, Nearly one-dimensional Hénon attractors and inverse limits, Nonlinearity 8 (1995), 29-42.

[7] M. Barge and J. Martin, Endpoints of inverse limit spaces and dynamics, in: Continua (Cincinnati, OH, 1994), Lecture Notes in Pure and Appl. Math. 170, Dekker, 1995, 165-182.

[8] M. Brown, Some applications of an approximation theorem for inverse limits, Proc. Amer. Math. Soc. 11 (1960), 478-483.

[9] K. Brucks and B. Diamond, A symbolic representation of inverse limit spaces for a class of unimodal maps, in: Continua (Cincinnati, OH, 1994), Lecture Notes in Pure and Appl. Math. 170, Dekker, 1995, 207-226.

[10] H. Bruin, Planar embeddings of inverse limit spaces of unimodal maps, Topology Appl. 96 (1999), 191-208.

[11] - Inverse limit spaces of post-critically finite tent maps, Fund. Math. 165 (2000), $125-138$.

[12] W. Dębski, On topological types of the simplest indecomposable continua, Colloq. Math. 49 (1985), 203-211.

[13] P. Collet and J. P. Eckmann, Iterated Maps on the Interval as Dynamical Systems, Birkhäuser, Boston, 1980.

[14] S. Holte, Generalized horseshoe maps and inverse limits, Pacific J. Math. 156 (1992), 297-305.

[15] L. Kailhofer, The partial classification of inverse limit spaces of tent maps with periodic critical points, Topology Appl. 123 (2002), 235-265.

[16] W. de Melo and S. van Strien, One-Dimensional Dynamics, Springer, New York, 1993.

[17] J. Mioduszewski, Mappings of inverse limits, Colloq. Math. 10 (1963), 39-44.

[18] S. Nadler, Continuum Theory, Dekker, New York, 1992.

[19] R. C. Swanson and H. W. Volkmer, Invariants of weak equivalence in primitive matrices, Ergodic Theory Dynam. Systems 20 (2000), 611-626.

[20] W. T. Watkins, Homeomorphic classification of certain inverse limit spaces with open bonding maps, Pacific J. Math. 103 (1982), 589-601.

[21] R. F. Williams, One-dimensional nonwandering sets, Topology 6 (1967), 473-487.

Department of Mathematics

Alverno College

3401 S 39th, Milwaukee, WI 53215, U.S.A.

E-mail: lois.kailhofer@alverno.edu 